\title{
Materiale Politisierung der Produktion
}

\section{Gesellschaftliche Herausforderung und institutionelle Innovationen in fortgeschrittenen kapitalistischen Demokratien}

\section{Herbert Kitschelt}

Department of Political Science, 214 Perkins Library, Duke University, Durham, North Carolina 27706, USA

$\mathrm{Z}$ u s a m m e $\mathrm{nf}$ a s sung: Die Modernisierungstheorien der sechziger Jahre ebenso wie eine rein historische Komparatistik sind unbefriedigend für eine makrosoziologische Theorie sozialen Wandels in fortgeschrittenen kapitalistischen Demokratien. Eine Neuformulierung der Erklärungslogik und Substanz von Entwicklungstheorien, die sowohl linearen Modernisierungs- als auch historistischen Annahmen entgeht, ist jedoch möglich (Abschnitt 1). Ihre Argumentationsstrategie läßt sich auf gegenwärtige gesellschaftliche Desintegrationsprobleme kapitalistischer Demokratien anwenden und mit alternativen Erklärungsansätzen politisch-ökonomischer Krisentheorien konfrontieren (Abschnitt 2). Abschließend können Forschungsperspektiven und empirische Hypothesen spezifiziert werden, die für dieses Theorienprogramm relevant sind (Abschnitt 3).

Ende der vierziger Jahre stellt der britische Soziologe T. H. Marshall die These auf, daß sich in der Entwicklung moderner kapitalistischer Gesellschaften eine Sequenz von politischen Institutionalisierungsschüben nachzeichnen ließe, die in der Ausbildung des Sozialstaates kulminiere. Der Sozialstaat, so Marshall, garantiere ein strukturelles Gleichgewicht zwischen den Sphären privatwirtschaftlicher Marktallokation und staatlicher Zuteilung von Lebenschancen. Ungleichheit in der Wirtschaftssphäre werde durch politische Institutionen (allgemeines Wahlrecht, soziale Sicherungssysteme und öffentliche Dienstleistungen) in einer Weise entgegengesteuert, da $\beta$ sich ein stabiles Gleichgewicht zwischen demokratischer Politik und kapitalistischer Marktwirtschaft einstelle (Marshall 1949).

Diese Beziehung zwischen Sozialstaat und kapitalistischer Wirtschaft ist seit Beginn der siebziger Jahre in den meisten fortgeschrittenen Industriegesellschaften aus dem von Marshall vorausgesagten Gleichgewicht geraten. Dieser Wandel gesellschaftlicher Integrationsbedingungen markiert, so die These dieser Arbeit, eine Wasserscheide in der Nachkriegsentwicklung des politisch-ökonomischen Weltsystems. Wirtschaftliches Wachstum, gefördert durch makro-ökonomische Staatsintervention, ermöglichte eine kontinuierliche Ausdehnung des Sozialstaates. Solche Entwicklungstrends lassen sich nun anscheinend nicht mehr umstandslos fortschreiben. Zugleich haben sich soziale Ansprüche mobilisiert, die nicht nur die Methoden, sondern auch die Zielregulative der Wachstumswirtschaft und des bürokratischen Sozialstaates in Frage stellen.
Auch wenn weithin Einigkeit über die grobe historische Periodisierung der Nachkriegsentwicklung besteht, gehen die Argumente jedoch weit auseinander, wenn die folgenden drei Fragen aufgeworfen werden:

(1) Worin genau besteht die Wasserscheide der siebziger Jahre, wenn man hinter die offensichtlichen Phänomene relativer wirtschaftlicher Stagnation, defizitärer Finanzhaushalte und „Verstopfung" des politischen Entscheidungsprozesses zurückgreift?

(2) Wie entsteht die neue Problemlage aus den Institutionen und Mechanismen sozialstaatlicher Massendemokratien?

(3) Welche wirtschaftlichen und politischen Handlungsoptionen bieten sich an, die neue Problemlage zu bearbeiten?

Die Gefährdung sozialstaatlicher Integrationskonzepte in einer seit dem zweiten Weltkrieg in entwickelten Demokratien ungewohnten Schärfe ist in zumindest zwei verschiedenen Theorieansätzen begriffen worden. Krisentheorien führen diese Entwicklung auf endogene politisch-ökonomische Trends in der Verteilung von funktionalen und personalen Einkommen zurück. Entwicklungstheorien behaupten demgegenüber, $\mathrm{da} \beta$ sich neue strukturelle Herausforderungen an die gegenwärtigen Gesellschaftssysteme eingestellt haben. Danach läßt sich die Geschichte heute entwickelter kapitalistischer Demokratien als irreversible Sequenz struktureller politischer und ökonomischer Problemschübe analysieren.

Die vorliegende Arbeit vertritt die These, da $\beta$ Entwicklungstheorien potentiell größere empiri- 
sche und theoretische Reichweite für die Erklärung des Problemdrucks in fortgeschrittenen Demokratien haben als Krisentheorien. Der erste Abschnitt skizziert das grobe Raster einer Entwicklungstheorie, die zwar an Überlegungen der funktionalistischen Modernisierungstheorie der sechziger Jahre anknüpft, aber zugleich weniger anspruchvoll sich der teleologischen, normativ apologetischen und unhistorischen Implikationen der älteren Entwürfe enthält. Im zweiten Abschnitt werden dann krisen- und entwicklungstheoretische Erklärungsvorschläge für die neuen gesellschaftlichen Herausforderungen der siebziger Jahre diskutiert, um zumindest die Bedingungen zu konkretisieren, unter denen die eine oder andere Theorie als empirisch gültig akzeptiert werden kann. Der Schlußabschnitt wirft Probleme und Hypothesen auf, die sich aus diesem Ansatz für weitere empirische Forschung ergeben.

\section{Sequenzen institutioneller Herausforderungen und Innovationen in kapitalistischen Gesellschaften}

In der modernen Makrosoziologie stehen sich „evolutionistische" Modernisierungstheorien und "historische" komparative Untersuchungen gegenüber (Brandt 1972). Die ersteren betonen interne gesellschaftliche Reifungsprozesse und interpretieren Modernisierung als quasi-teleologischen Pro$z e B$ der Entfaltung gesellschaftlicher Anpassungskapazitäten, welche ihre höchste Ausprägung in kapitalistischen Demokratien erreichten (vgl. Parsons 1964; 1966). Historische Ansätze haben demgegenüber auf die vielfältigen Variationen des Entwicklungsprozesses von Industriegesellschaften hingewiesen. Diffusion, Timing, internationale Konfigurationen und Koalitionen sozialer Gruppen sind ihre zentralen Konzepte (Bendix 1977: bes. 404-434; Moore 1966; Smith 1973; Appleby 1978; Gourevitch 1978).

So zutreffend die Kritik des Historismus am herkömmlichen Evolutionismus ist und so subtil seine historischen Analysen oft ausfallen, so wenig läßt sich jedoch bestreiten, daß auch ihm zumindest ein Idealtypus gleichsinniger Entwicklung "moderner“ kapitalistischer Industriegesellschaften zugrunde liegt und er somit neben Differenzen zwischen solchen Gesellschaften auch ihre strukturellen Gemeinsamkeiten anerkennt. Diffusionserklärungen gesellschaftlichen Wandels allein sind schon deshalb unbefriedigend für eine Erklärung solcher Gemeinsamkeiten, weil sie Strukturen und Prozes- se voraussetzen müssen, die gegenüber dem, was diffundiert wird, rezeptiv sind.

Die Auseinandersetzung zwischen den beiden Theoriegruppen spitzt sich deshalb auf die Frage $\mathrm{zu}$, wie stark evolutionistischen Annahmen sein dürfen, die in Entwicklungsmodelle eingehen. Hier soll eine "schwache“ Entwicklungstheorie vertreten werden, die keinen notwendigen, universellen Evolutionsprozeß annimmt, sondern Entwicklungsbedingungen strukturell relativiert. Sofern in verschiedenen, voneinander relativ autonomen Sozialsystemen ähnliche strukturelle Anfangsbedingungen vorliegen und sofern ihnen bestimmte strukturelle und kulturelle Parameter im Transformationsprozeß gemeinsam bleiben, werden sie mit ähnlichen Integrationsproblemen konfrontiert werden und ähnliche Ergebnisse sozialen Wandels zeitigen. Eine Reihe historischer Transformationen wird kontingenztheoretisch auf gemeinsame Strukturprämissen zurückbezogen. Diese stellen "funktionale Voraussetzungen“ für bestimmte Entwicklungsoptionen dar. Die Beibehaltung solcher Strukturprämissen ist weder notwendig noch „evolutionär überlegen“. Jede neue Herausforderung an die Integrationskapazität von Gesellschaften stellt auch einen ,choice point" dar, an dem solche grundlegenden Prämissen zur Disposition stehen und Gesellschaften durch ihre Revolutionierung aus einer Entwicklungssequenz ,aussteigen" können. Es gibt keine übergreifende, notwendige, endogene Entwicklungslogik, die ohne strukturelle Kontingenzen definierte, was ,progressive" und „regressive" Entwicklungen sind.

Innerhalb solcher methodologischer Grenzen sucht eine Entwicklungstheorie moderner kapitalistischer Demokratien (a) Organisationsprobleme zu erfassen, die sich ihnen gleichermaßen in ihrer Entwicklung gestellt haben, und (b) zumindest idealtypisch eine Abfolge oder "Sequenz" solcher Problemschübe nachzuzeichnen und zu erklären. Von historisch-konkreten Abweichungen einzelner Länder muß dabei zuweilen abstrahiert werden. Indem die Theorie jedoch nur anstrebt, idealtypisch Entwicklungsprämissen in den heutigen „Kern"staaten des kapitalistischen Weltsystems, deren Herausdifferenzierung zwischen dem 15. und 18. Jahrhundert einsetzte (Wallerstein 1974) $\mathrm{zu}$ beleuchten, befreit sie sich von dem überzogenen Anspruch der Modernisierungstheorien, allgemeine soziale Evolutionsbedingungen $\mathrm{zu}$ benennen.

Im Rahmen des so historisch eingegrenzten Untersuchungsuniversums ist es immer noch sinnvoll, 
formell souveräne Staaten als Ausgangspunkt und analytische Einheiten von Entwicklungsprozessen zu wählen. Inwieweit zunehmend komplexe Interdependenz zwischen Nationalstaaten im Weltsystem diese Bestimmung der Untersuchungseinheit in Zukunft sinnlos machen könnte, bleibt offen. Vorläufig ist die Relevanz einzelstaatlicher Einheiten im Entwicklungsproze $ß$ zugleich geringer und größer geworden, $d$. h. territoriale Differenzierung hat ihre Bedeutung kaum eingebüßt (vgl. Keohane/Nye 1977; Hanrieder 1978). Alle Anwendungsfälle der angestrebten strukturell relativierten Entwicklungstheorie teilen die Bedingung, Märkte durch Kommodifizierung von Produktionsfaktoren und -resultaten zum Basisparameter oder zentralen gesellschaftlichen Koordinationsmechanismus gemacht zu haben. Länder, die diesen Parameter aufheben (etwa durch umfassende zentrale Planung des Wirtschaftsprozesses) können nicht als Anwendungsfall der spezifischen Theorie betrachtet werden.

Des weiteren muß jede Entwicklungstheorie die Einheit von Entwicklungsperioden ebenso wie qualitative "Periodenbrüche“ bestimmen können, die kritische „choice points" markieren. Dies ist häufig mit einem punktuellen Krisenbegriff versucht worden, in welchem Krisen plötzliche soziale Koordinationsprobleme (Krisen der Systemintegration) und soziale Bewegungen bezeichnen, die sich gegen zentrale gesellschaftliche Institutionen wenden (Krisen der Sozialintegration). Ein punktueller Krisenbegriff macht jedoch, wie historische Arbeiten gezeigt haben (Grew 1978), das Auffinden von Entwicklungssequenzen zu unübersichtlich und schwierig. In einer makrosoziologischen Theorie ist es sinnvoller, Entwicklungsperioden mit dem Auftreten institutioneller Innovationen zu identifizieren (Verba 1971: 298) und „Periodenbrüche" als Zeiträume zu verstehen, in denen wiederholte Krisen fundamentale system- und sozialintegrative Instabilitäten anzeigen. Eine Theorie der Periodenbrüche muß deshalb auf Generationsmechanismen von Krisen, nicht punktuelle Krisen, abstellen (vgl. dazu Offe 1973). Und die Einführung von institutionellen Innovationen vollzieht sich zumeist als iterativer und interdependenter ProzeB, in welchem soziale Mechanismen, die Krisendruck erzeugen, nicht ein für allemal ,aufgelöst" ", sondern wiederholt bearbeitet werden (Grew 1978a: 14). Periodenbrüche sind deshalb nicht mit Revolutionen, d. h. raschem sozialem und politischem Wandel gleichzusetzen, sondern zeigen Kräfte an, die auf eine qualitativ neue Entwicklungsrichtung sozialer Institutionen drängen.
Schließlich muß eine Entwicklungstheorie Problemdimensionen angeben können, auf denen $\mathrm{Ge}$ sellschaften institutionelle Innovation erfahren. Universelle funktionalistische Entwicklungstheorien der sechziger Jahre identifizierten als solche Dimensionen die progressive Ausdehnung sozialer Differenzierung, politischer Steuerungskapazität und gesellschaftlicher Gleichheit (Binder 1971: 17ff.; Coleman 1971). Für die Zwecke einer historisch bescheideneren Theorie können diese Entwicklungsdimensionen durch Anleihen aus der marxistischen Kapitalismustheorie (,wirtschaftliche Differenzierung"), aus Stein Rokkans politischer Soziologie (,politische Kapazitätssteigerung") und T. H. Marshalls Rekonstruktion des Sozialstaates („Gleichheit“) präzisiert werden. Eine solche historische Konkretisierung kann auch eine einfache lineare Änderung der Variablenwerte auf den einzelnen Dimensionen (,mehr“ Differenzierung, Kapazität, Gleichheit) als Entwicklungsschema vermeiden und vielmehr strukturelle und qualitative Innovationen auf den einzelnen Dimensionen benennen.

(1) In der marxistischen Kapitalismustheorie ist die ökonomische Entwicklung als Phasenabfolge von Produktionsverhältnissen und Produktivkräften erfaßt worden ${ }^{1}$. Eine fünfstufige Entwicklungssequenz läßt sich dabei als vielleicht kleinster gemeinsamer theoretischer Nenner für die Entwicklung kapitalistischer Gesellschaften herausschälen, ohne interne Kontroversen der marxistischen Historik zu berücksichtigen: Der Auflösung des Feudalismus (a) folgte eine Phase merkantiler Produktionsverhältnisse, einfacher Warenproduktion und frühkapitalistischer Manufaktur (b). Eine vollständige Kommodifizierung aller Produktionsfaktoren erreicht ihren Höhepunkt im Konkurrenzkapitalismus (c). Die beiden jüngeren Phasen kapitalistischer Entwicklung weisen eine zunehmende Vermittlung marktwirtschaftlicher Allokation durch formale Organisation auf, zunächst im Monopolkapitalismus (d) durch Selbstorganisation von Kapital und Arbeit, dann im politischen Kapitalismus (e) durch mikro- und makro-ökonomische Intervention des Staates in die Marktallokation (vgl. Shonfield 1965; O'Connor 1973). Zentral für die jüngste Problematik systemischer Krisenerscheinungen ist dabei die Tatsache, daß auch im politi-

\footnotetext{
${ }^{1}$ Vgl. zur Periodisierung des Kapitalismus besonders Poulantzas 1975: Teil II; als historische Studien Dobb 1946; Wallerstein 1974; Anderson 1974; Hilton 1976 und kritisch Elsenhans 1983.
} 
schen Kapitalismus die marktwirtschaftliche Produktion nur formal politisiert wird, indem wirtschaftliche Aufgaben zwar politisch-administrativ behandelt werden, ohne jedoch die Rationalitätskriterien privaten Marktverhaltens (Allokationseffizienz, Kapitalakkumulation, Rentabilität) anzutasten. Politische Organisation fügt sich sozusagen einem „Subsidiaritätsprinzip", in welchem Markteffizienz Vorrang behält.

(2) Nicht-marxistische Entwicklungstheorien moderner politischer Systeme haben sich, nach einigem Experimentieren, auf vier Problemfelder institutioneller Innovation einigen können, die für die europäische Entwicklung insbesondere von Stein Rokkan in vergleichenden Analysen bemüht worden sind (vgl. Rokkan 1970: 61ff.; 1975: 565-573; sekundär Flora 1981). Als erste Schwelle ist dabei die Bildung von Flächenstaaten mit institutionellen Innovationen wie stehendem Heer, rationalisierter Bürokratie und regelmäßiger Steuererhebung verknüpft. Diese ursprünglich auf purem Zwang beruhenden Gebilde (vgl. Tilly 1975) steigerten ihre soziale Integrationskraft und Handlungsfähigkeit durch organisatorische und ideologische Innovationen, die mit der Nationenbildung zusammenhängen. Kulturell-normative ,nationale“ Identitäten bilden sich um die territorialen Herrschaftsgebiete und stehen in engem Wechselverhältnis mit einer Reihe institutioneller Korrelate: formelle zivile Bürgerrechte (insbesondere Eigentumsschutz) und politische Verfassungen, allgemeine Wehrpflicht, staatliches Schulsystem, Unterordnung unter und/oder Trennung religiöser Organisationen vom Staat. Eine dritte Innovationsschwelle bildet die Durchsetzung politischer Partizipationschancen der aus ,Untertanen“ in „Staatsbürger" sich verwandelnden Gesellschaftsmitglieder durch Wahl einer Volksvertretung, die dann auch die Verantwortlichkeit der Regierung gegenüber und schließlich die Regierungsbildung durch die Volksvertretung durchsetzt. Die formale Gleichheit aller Staatsbürger in politischen Beteiligungsrechten bereitet ihrerseits als vierte Innovation Anstrengungen zur Herstellung materialer Gleichheit durch sozialstaatliche Institutionen vor, welche die einzelnen vor existentiellen Risiken der Marktvergesellschaftung schützen und Minimalbedingungen sozialer Gleichheit erfüllen.

(3) Im politisch-moralischen Diskurs kann die gleiche Sequenz von institutionellen Entwicklungsschüben aus der Sicht der Herrschaftsadressaten als Mobilisierung qualitativ verschiedenartiger Ansprüche erscheinen, die durch Zugeständnisse der
Eliten beantwortet werden. Nach Marshall (1949) wurde mit der Auflösung vorkapitalistischer Sozialordnungen auch das Netz traditionaler wirtschaftlicher, politischer und sozialer Bürgerrechte im ProzeB der Bildung von Zentralstaaten zerschlagen. Solche Rechte wurden mit der Entwicklung von bürgerlicher Gesellschaft und Politik durch neue Formen und Inhalte sequentiell rehabilitiert: Zunächst erfolgte ein Kampf um ,passive“ bürgerliche Freiheiten, der sich auf Eigentumsschutz, faire Rechtsverfahren, Religionsfreiheit und freie Meinungsäußerung bezog. Sodann entfachte sich eine Auseinandersetzung um ,aktive“ staatsbürgerliche Beteiligungsrechte an der Politik. Schließlich konzentrierte sich die Debatte auf „soziale" Bürgerrechte mit Minimalstandards wirtschaftlicher Versorgung.

(4) Im Anschluß an Rokkan (1970) kann eine recht enge, wenn auch empirisch nicht immer präzise Korrespondenz zwischen der Ausbildung politischer Kapazitäten einerseits und typischen gesellschaftlichen Konfliktlinien andererseits, um die sich soziale Akteure im Kampf für institutionelle Innovationen mobilisierten, beobachtet werden. Die Auflösung vorkapitalistischer Rechtsverhältnisse und Identitätsbindungen in der Staats- und Nationenbildung waren mit einem Kampf zwischen Zentrum und Peripherie, hergebrachten lokalen und neuen zentralen Herrschaftsinstanzen, ebenso verbunden wie mit einer Auseinandersetzung zwischen Staat und Kirchen um säkulare oder religiöse politische Identitätsbindung. Die Forderung breiter politischer Beteiligungsrechte stellte die industriellen Klassen (zunächst das Bürgertum, dann zunehmend die Arbeiterklasse) den Agrareliten entgegen, die den Staatsapparat in der Vergangenheit kontrolliert hatten. Und die Ausbildung des Sozialstaates schließlich wird recht trennscharf entlang den Konfliktlinien des Klassengegensatzes im Kapitalismus ausgefochten. Die vier Entwicklungsdimensionen moderner kapitalistischer Demokratien sind in Schema 1 dargestellt. Zum einen ergänzt dieses Schema schon im Vorgriff auf die weitere Diskussion dieses Aufsatzes das Schema von politischen Rechtsforderungen sozialer Akteure und gesellschaftliche Konfliktachsen um Problemlagen, die hier als materiale Politisierung der Produktion diskutiert werden sollen. Entwicklung wird damit als offener, nicht teleologisch geschlossener Prozeß begriffen.

Zum anderen soll dieses Schema im Gegensatz zu vorliegenden Entwicklungstheorien, die sich jeweils auf eine der Dimensionen konzentrieren, das 
Schema 1 Strukturen und Entwicklungssequenzen fortgeschrittener kapitalistischer Demokratien

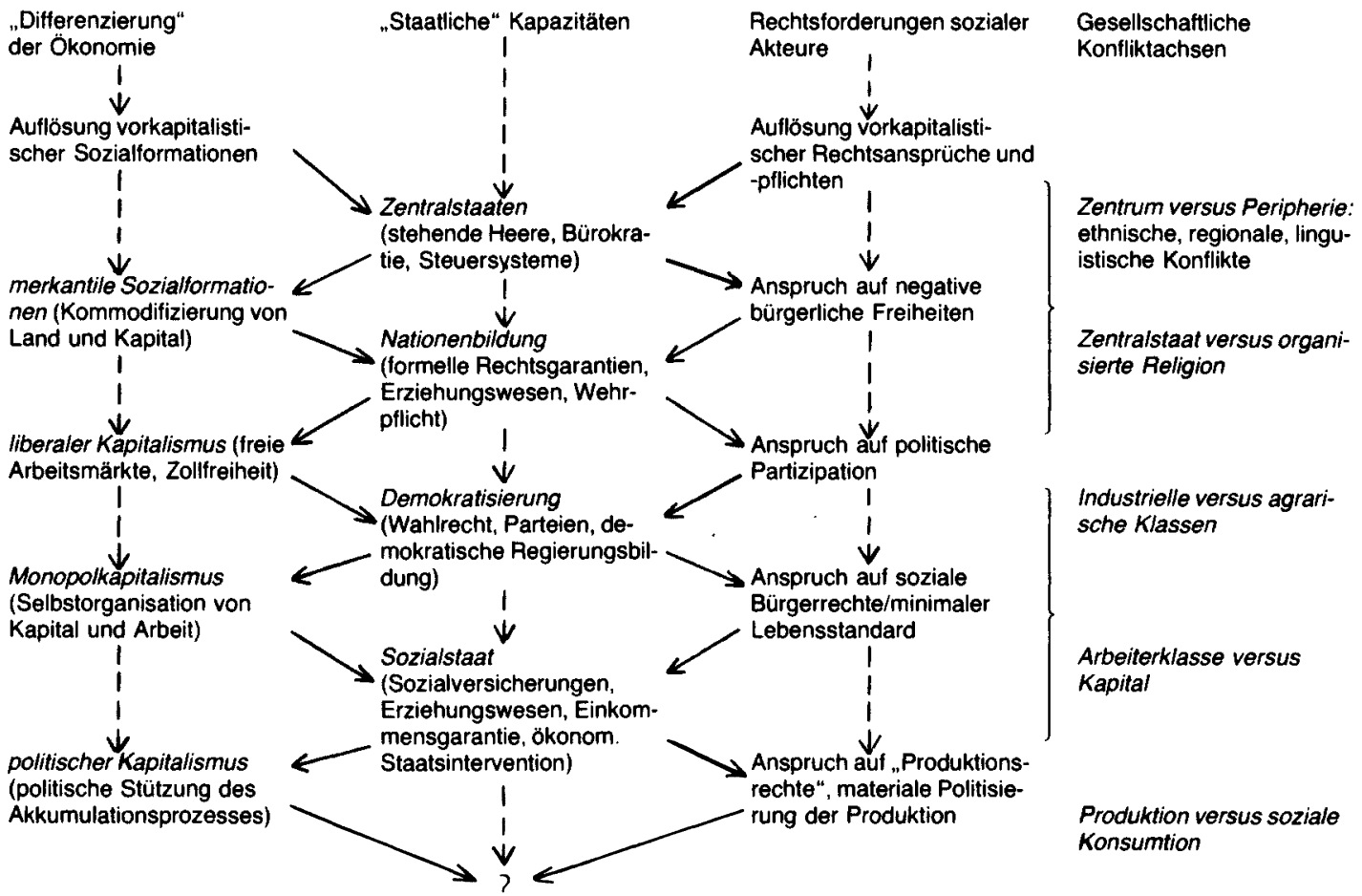

dynamische Wechselspiel zwischen ökonomischer Akkumulation, staatlicher Kapazitätsausbildung, politisch-moralischen Ansprüchen und gesellschaftlichen Konfliktachsen nahelegen. Der Ökonomismus marxistischer Stufenmodelle, in welchen politische und kulturelle Bedingungen zumeist als abhängige Variablen erscheinen (vgl. Autorengruppe 1981; Hobsbawm 1981; Thompson 1983) und politische Regimeinnovationen als Konsequenz ökonomischer Produkt- und Produktionszyklen auftreten (Kurth 1979a und b), kann damit ebenso relativiert werden wie der komparativ-statische und auf politische Eliten zentrierte Charakter von Rokkans Theorie (vgl. kritisch Alford/ Friedland 1974) ${ }^{2}$. Vielmehr legt das Schema einen interaktiven Prozeß nahe, in welchem wirtschaftliche Entwicklungen und politische Mobilisation von neuen Ansprüchen zwar gesellschaftlichen Transformationsdruck erzeugen, jedoch erst institutionelle Innovationen die Überwindung desinte-

${ }^{2}$ Flora (1981: 401) erklärt den komparativ-statischen Charakter von Rokkans Entwicklungstheorie aus dessen Interesse an historischer Variation zwischen den einzelnen westeuropäischen Ländern. grativer Krisen erlauben. Neue institutionelle Steuerungskapazitäten ermöglichen ihrerseits eine erneute Transformation von Ökonomie und soziokulturellen Ansprüchen ${ }^{3}$.

Erst die Ausbildung von Flächenstaaten erlaubte die Enfaltung des merkantilen Frühkapitalismus und ein Programm passiver Bürger- und Eigentumsrechte, welches im Zentralstaat seinen Adressaten fand ${ }^{4}$. Die Innovation des Nationalstaates

${ }^{3}$ Eine ähnliche Erklärungslogik wird von Habermas (1976: 233f.) im Rahmen einer ,starken“ Entwicklungstheorie verfolgt, deren Ansprüche allerdings über diejenigen der hier skizzierten Sequenztheorie weit hinausgehen, weil sie einen universellen, nicht strukturell kontingenten Gültigkeitsanspruch erhebt und normative Evaluationskriterien sozialen Wandels impliziert (vgl. kritisch Schmid 1981: 29).

${ }^{4}$ Die Bedeutung politischer Institutionen und Ideologien für die wirtschaftliche Entwicklung wird auch in Arbeiten der Neuen Wirtschaftsgeschichte betont: vgl. North/Thomas 1973: Kap. 8-12; North 1981: Kap. 3, 11; siehe auch allgemeiner Gerschenkron 1962; Gilpin 1975. 
verlieh Forderungen nach politischer Partizipation mehr argumentativen Zwang und erleichterte zugleich die Entwicklung eines liberalen Kapitalismus, welcher nicht durch partikulare Kollektividentitäten, sondern bürgerlichen Individualismus und Nationalismus gekennzeichnet war. Die Durchsetzung des allgemeinen Wahlrechts und die Entwicklung von bürokratisierten politischen Parteien wiederum beförderte eine Kollektivierung des gesellschaftlichen Lebens, welcher sowohl Entwicklungen im Wirtschaftssystem (industrielle Großorganisationen, Spitzenverbände der Produzentenklassen) als auch dann sozialpolitische Ansprüche korrespondierten. Der Sozialstaat resultiert aus einem Kompromiß zwischen den gesellschaftlichen Hauptklassen im Kapitalismus. Seine Stärke variiert deshalb nicht zuletzt mit dem industriellen und politischen Organisationsgrad von Gewerkschaften, Unternehmern und politischen Parteien, die sozio-ökonomische Konfliktlinien repräsentieren (vgl. Cameron 1978; Castles 1978; Schmidt 1982).

Auch ein Interaktionsmodell verschiedener Entwicklungsdimensionen löst den Anspruch auf eine dynamische Theorie sozialen Wandels nicht vollständig ein. Verschiedene Ansätze in der komparativen Politikanalyse haben beobachtet, da $\beta$ in Phasen relativer gesellschaftlicher Stabilität politische Strukturen in Systemen mit ähnlichen Basisparametern zur Konvergenz tendieren. Dagegen "explodiert" die Variation politischer Strategien in Zeiten neuer gesellschaftlicher Herausforderungen und Integrationsprobleme, wenn die Spielregeln politischer und ökonomischer Integration selbst Konfliktgegenstand werden (vgl. Gourevitch 1984; Kitschelt 1983a: 56ff.; Krasner 1984: 240ff.). Politisch-institutionelle Regimeformen (Katzenstein 1978) und/oder neue Koalitionen gesellschaftlicher Kräfte (Gourevitch 1977; 1984) beeinflussen die spezifischen Prozesse der Problembewältigung in einzelnen Ländern. Entwicklungstheorien insistieren aber darauf, $\mathrm{da} B$ Gesellschaften mit ähnlichen Basisparametern nach einer "trial and error" $\mathrm{Pe}$ riode politischer Innovation ähnliche (oder funktional äquivalente) Institutionen zur Bearbeitung ähnlicher Desintegrationserscheinungen auf Dauer stellen werden. Ein solches Theoriemodell, welches sich die Unterscheidung von Variations-, Selektions- und Stabilisierungsprozessen als „,evolutionäres" Entwicklungsschema zu Nutze macht, braucht keine teleologische Erklärung sozialen Wandels anzunehmen. Kriterien und Methoden erfolgreicher Innovation schälen sich in trail-anderror Prozessen gesellschaftlichen Lernens, die durch strukturelle Ausgangsbedingungen begrenzt sind, nach einer teleonomischen Logik (Van Parijs 1982) heraus 5 .

Aber auch innerhalb eines solchen Modells bleibt die Erklärungskraft einer Entwicklungstheorie limitiert. Nicht in allen Fällen westeuropäischer Staatenbildung wird die idealtypische Sequenz von institutionellen Innovationen in der angegebenen Reihenfolge eingelöst (vgl. Flora/Albers 1981 und Grew 1978a: 29). Außerdem müßte eine komplexere Theorie historischer Prozesse das Timing von Herausforderungen in einzelnen Ländern und ihre Lokalisierung im politisch-ökonomischen Weltsystem berücksichtigen ${ }^{6}$.

Die weitaus schwerwiegendste Begrenzung bisheriger Entwicklungstheorien mag jedoch darin bestehen, daß sie die Entwicklungssequenz moderner Demokratien tendenziell mit einer teleologischen Abschlußhaftigkeit darstellten. Dies war solange nicht bloß Ausdruck politisch-apologetischer Realitätsblindheit, als prinzipiell neue gesellschaftliche Herausforderungen weitgehend jenseits des empirischen Wahrnehmungshorizontes der Sozialforschung und der sozialen Alltagserfahrung lagen. Nun besteht die These dieser Arbeit jedoch darin, $\mathrm{da} ß$ sich seit den siebziger Jahren in entwickelten kapitalistischen Ländern ein neuer historischer Periodenbruch, $d$. $h$. eine zunehmende Inkompatibilität zwischen den existierenden politisch-ökonomischen Institutionen und den Integrationsbedingungen moderner Gesellschaften, anbahnt. Diese sich erst umrißhaft abzeichnende Lage soll im Anschluß an Ronges (1975) Begriffsbildung als ,,materiale Politisierung der Produktion" in einem doppelten Sinne bezeichnet werden.

Zum einen ist es aus der Perspektive der Systemintegration kapitalistischer Demokratien schwierig geworden, die institutionellen Bedingungen $\mathrm{zu}$ spezifizieren, unter denen effiziente Marktallokation und Akkumulation sich vollziehen können. Dies gilt, wie im nächsten Abschnitt illustriert wird, sowohl für die Marktteilnehmer selbst als auch für politische Institutionen, denen Hand-

\footnotetext{
${ }^{5}$ Diese Überlegungen knüpfen insofern an Luhmanns Evolutionstheorie an, als sie auch eine „Kontingenzkausalität" und "strukturelle Unterdetermination" im historischen Prozeß annehmen. Entwicklungsmöglichkeiten bestimmen sich situativ, nicht nach einem übergreifenden teleologischen Schema (vgl. Luhmann 1975: 156f., 162).

${ }^{6}$ Dies wird - wenn auch in ökonomistisch-reduktionistischer Weise - von Kurth 1979a angestrebt.
} 
lungskriterien fehlen, politischen Interventionismus an Standards wirtschaftlicher Allokationseffizienz und wirtschaftlichem Wachstum zu orientieren (Offe 1974).

Zum anderen ist es aus der Perspektive der Sozialintegration heutiger Demokratien für bedeutende Bevölkerungsteile unplausibel geworden, die existierenden wirtschaftlichen und politischen Institutionen für legitim zu halten, weil die formale Rationalität des kapitalistischen Wirtschaftsprozesses mit den materialen Ansprüchen auf steigende Lebenschancen konvergiere. Für solche Gruppen ist die Steigerung von Wohlstand (i. S. monetärer Kaufkraft) und Wohlfahrt (i. S. sozialer Bedürfnisbefriedigung) nicht mehr ein gleichsinniger Prozeß, sondern vielmehr ein Nullsummenspiel. Während Märkte und politische Prozesse sich auf die Steigerung von Wohlstand und die vertikale Verteilung von Lebenschancen durch Einkommen und Bedingungen politischer Partizipation spezialisiert haben, werden soziale Bedürfnisse systematisch benachteiligt, die solchen Kriterien nicht gehorchen. Es kommt zu einer horizontalen Disparität zwischen den organisierten Interessen an Einkommensansprüchen, die im "produktiven Kern“ des politischen Kapitalismus angesiedelt sind, und solchen, die sich organisatorisch an seiner Peripherie befinden und nicht durch Einkommenszuteilungen befriedigt werden können ${ }^{7}$.

Mit einiger definitorischer Vorsicht kann man die neue Konfliktachse, die sich um die materiale Politisierung der Produktion zentriert, als Konflikt zwischen „Produktion" und „Konsumtion“ bezeichnen (vgl. Touraine 1973; 159ff.). Produktiv sind Zielverzichte und Leistungen, die Optionen zur Bedürfnisbefriedigung herstellen, ohne selbst konsumtive Qualitäten aufweisen (z. B. Geldeinkommen). Konsumtiv sind solche Aktivitäten und Ressourcen, die unmittelbar ihren Selbstzweck in der Bedürfnisbefriedigung haben. Die Begrenzung dessen, was zu Produktion oder Konsumtion gehört, ist eine Frage gesellschaftlicher Organisation. Durch Aufkommen der Fabrik- und Büroorganisation industrieller Gesellschaften z. B. ist „Arbeit“" weitgehend aus einem Selbstzweck in einen Zielverzicht umdefiniert worden, welchem intrinsische

${ }^{7}$ Es ist kein Zufall, daß diese These in den sechziger Jahren während der Hochkonjunktur der Studentenbewegung entwickelt wurde, welche zum ersten Mal auf eine materiale Politisierung der Produktion und Kritik der Wachstumsgesellschaft hinarbeitete: Bergmann et al. 1968; Offe 1969; Touraine 1971.
Befriedigung entgeht. Arbeit wird in dem Maße eine „konsumtive“ Aktivität, als sie von Zwecken intrinsischer Gratifikation gesteuert wird. Die materiale Politisierung der Produktion stellt nun zur Debatte, welche Aktivitäten und Ressourcen gesellschaftlich nach produktiven oder konsumtiven Kriterien organisert werden sollen, und fragt, ob die Tendenz kapitalistischer Gesellschaften, immer mehr Güter, Ressourcen und Fähigkeiten nach produktiven Kriterien $\mathrm{zu}$ bewerten, umgekehrt werden soll. Im Vokabular der älteren marxistischen Sozialtheorie ist dieses Problem als Spannung zwischen tauschwert- und gebrauchswertorientiertem sozialem Prozeß beschrieben worden, bei Max Weber als Widerspruch zwischen der formalen Rationalität und materialen Irrationalität des Kapitalismus.

Als empirische Referenz für eine materiale Politisierung der Produktion lassen sich zwei Beobachtungen heranziehen. Unter systemintegrativen Gesichtspunkten sind entwickelte kapitalistische Gesellschaften seit Beginn der siebziger Jahre in eine Phase relativer wirtschaftlicher Stagnation eingetreten, die durch steigende Arbeitslosigkeit, geringes Wirtschaftswachstum und Inflation gekennzeichnet ist. Unter sozialintegrativen Gesichtspunkten sind seit Ende der sechziger Jahre eine Reihe von sozialen Bewegungen, wie etwa Studenten-, Ökologie- und Frauenbewegungen, aufgetreten, die eine vornehmlich „produktive“ Nutzung von Gütern, Ressourcen und Fähigkeiten im Kapitalismus in Frage stellen. Diese Forderungen betreffen ein so weites Feld von Themen wie die Organisation von Arbeit und Ausbildung, die wirtschaftliche Nutzung von Natur und die Kommerzialisierung von Sexualität, um nur einige wichtige Beispiele zu nennen ${ }^{8}$.

Das Problem einer Entwicklungstheorie, die neue system- und sozialintegrative Herausforderungen kapitalistischer Demokratien aus der Interferenz zwischen produktiven und konsumtiven Nutzungsweisen natürlicher und sozialer Ressourcen herleitet, besteht jedoch darin, sich mit einer Reihe von Krisentheorien auseinandersetzen zu müssen, die die gleichen desintegrativen Merkmale moderner Gesellschaften in anderer Weise als Interferenztheorien erklären. In Krisentheorien stehen dabei als erklärende Variablen wirtschaftliche Verteilungskämpfe zwischen Weltregionen, Produktions-

\footnotetext{
${ }^{8}$ Vgl. Melucci 1980; 1981; Touraine 1977; 1978; Moscovici 1977; empirisch: Brand/Büsser/Rucht 1983; Brand (Hg.) 1985;
} 
Schema 2 Alternative Erklärungsmuster gesellschaftlicher Desintegration

1. Krisentheorien

Logik des politischen Kapitalismus

neue Qualität der funktionellen Einkommensverteilung (Redistribution zugunsten der Arbeit)

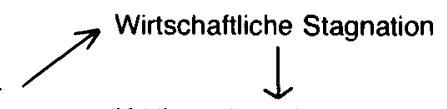

Kritik an der existierenden Wirtschaftsordnung

\section{Interferenztheorien}

Logik des politischen Kapitalismus

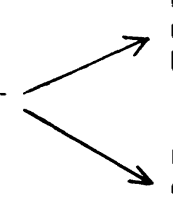

neue Qualität der Investitions und Akkumulationsmöglichkeiten

materiale Politisierung der Produktion, Kritik an der existierenden Wirschaftsordnung sektoren, Produzentenklassen und Klassenfraktionen im Vordergrund. Solche Analysen nehmen an, daß weiterhin ein universeller Konsens über die Ziele des Wirtschaftsprozesses besteht (Wachstum, monetäre Einkommen, Vollbeschäftigung, Geldwertstabilität, usw. $)^{9}$ oder zumindest eine materiale Politisierung der Produktion nur als Folge wirtschaftlicher Stagnation, nicht als unabhängig koexistierendes Phänomen oder gar als eine Ursache wirtschaftlicher Stagnation, verstanden werden kann. Verteilungsprobleme, nicht Fragen der Kapitalakkumulation selbst und der Motivationsbasis kapitalistischer Wachstumsgesellschaften, stehen im Mittelpunkt von Krisentheorien.

Die Unterschiede zwischen der Erklärungslogik von Krisen- und von Inteferenztheorien für aktuelle gesellschaftliche Integrationsprobleme sind vereinfacht in Schema 2 skizziert. Es kommt nun darauf an, die Gründe zu prüfen, mit denen die Validität der konkurrierenden Erklärungsmodelle verteidigt wird. Ein solcher Test kann im Rahmen des vorliegenden Aufsatzes noch nicht nach strikten empirischen Kriterien erfolgen. Vielmehr sollen vorläufig nur Argumente zusammengestellt werden, die in dieser Debatte eine Rolle spielen. Empirische Sachverhalte werden dabei illustrativ, nicht systematisch, herangezogen, um die Geltungsansprüche von Interferenztheorien tatsachennäher darzustellen. Vielleicht kann damit zumindest ein Beitrag zur Klärung der Bedingungen

\footnotetext{
${ }^{9} \mathrm{Vgl}$. so etwa explizit Heidenheimer et al. 1983: 124. Ein ähnlicher praktischer und analytischer Bias findet sich auch in den Arbeiten von Schmidt 1982 und Zysman 1983.
}

geleistet werden, unter denen eine der Konkurrenten für empirisch plausibler gehalten werden mag als die andere.

\section{Verteilungskrise oder materiale Politisierung der Produktion?}

Interferenztheorien können die Validität von Krisentheorien über moderne kapitalistische Demokratien dann erfolgreich angreifen, wenn sie die folgenden Argumentationsziele einlösen. (1) Die von Krisentheorien angenommene Ursachen für wirtschaftliche Stagnation und politische Konflikte variieren nicht empirisch mit der Ausprägung von Krisen in einzelnen Ländern. (2) Interferenztheorien können bestimmte Stagnationsphänomene genauso gut oder besser als Krisentheorien erklären. (3) Auf Krisenerklärungen bauende politische $\mathrm{Re}$ formstrategien konfligieren mit sozialen Protestpotentialen, die nur von Interferenztheorien erklärt werden. (4) Kritik an der Systemlogik des politischen Kapitalismus läßt sich nicht ursächlich auf dessen Performanzschwächen, sondern gerade eher auf dessen Erfolg bei der Gestaltung wirtschaftlicher und politischer Institutionen beziehen. Vor dem Hintergrund dieser argumentativen Spielregeln lassen sich nun fünf zentrale Argumente der Krisentheorien kritisch diskutieren. Darauf folgt eine Skizze von fünf bislang empirisch weit weniger sorgfältig bearbeiteten Thesen der Interferenztheorien.

(1) Die vielleicht prominenteste These von rechten und linken Krisentheoretikern macht Verschiebungen der Faktoreinkommen zugunsten der Arbeit und auf Kosten des Kapitals für die Krise der 
siebziger Jahre verantwortlich. Nun sind zwar Profitraten in allen kapitalistischen Ländern in den vergangenen Jahrzehnten gesunken ${ }^{10}$. Aber dies erklärt kaum, warum ähnlich hohe Profitraten (in den USA, der Bundesrepublik, Japan) mit sehr unterschiedlichen Wachstumsraten einhergingen oder auch extrem niedrige Profitraten dennoch mit im internationalen Vergleich nur wenig niedrigeren Wachstumsraten zusammenfielen (z. B. in Schweden).

Auch im longitudinalen Vergleich ist die Verschiebung von Faktoreinkommen kaum ein gutes Argument, um Krisenerscheinungen zu erklären. Der immer wieder behaupteten Lohnexplosion der siebziger Jahre ${ }^{11}$ steht der Sachverhalt gegenüber, $\mathrm{da} ß$ die bereinigte Lohnquote in allen entwickelten kapitalistischen Ländern am Ende der siebziger Jahre kaum höher war als zu Beginn des Jahrzehnts (Glastetter et al. 1983: 352). Zugleich fielen die Bruttoinvestitionsquoten am Bruttosozialprodukt in den siebziger Jahren so geringfügig, da $B$ sie 1981 kaum unter dem Stand von 1970 lagen (World Bank 1982: 10). Und der Anteil staatlicher Investitionen fiel zugunsten konsumtiver (Sozial)ausgaben nur in Ausnahmefällen (Kohl 1981).

Eine Reihe von Krisentheorien hat argumentiert, $\mathrm{da} ß$ eine starke Repräsentation der Arbeiterbewegung in der Politik durch zentralistische, mitgliederstarke Gewerkschaften, mächtige sozialdemokratische Parteien und deren Regierungsbeteiligung eine Institutionalisierung von Klassenbeziehungen fördere, die ihrerseits mit relativ besserer ökonomischer Performanz zusammenfiele (vgl. Schmitter 1981; Castles 1978; 1982; Schmidt 1982; Lange/Garrett 1985). Auch wenn solche Untersuchungen erfolgreich sind, Variationen von Inflationsraten und Arbeitslosigkeit zwischen entwikkelten kapitalistischen Demokratien zu beleuchten, liefern sie bislang wenig Ansatzpunkte, Variationstrends des Wirtschaftswachstums zu erklären.

Wenn globale verteilungspolitische Krisentheorien unbefriedigend bleiben, mögen vielleicht sektorale und mikroökonomische Analysen weiterhelfen.

${ }^{10}$ Einschlägige Datenzeitreihen finden sich in T. P. Hill, Profits and Rates of Return (Paris, OECD 1979: 124), zitiert nach Semmler 1982: 33.

11 Für konservative Analysen vergleiche Freeman 1981; Emminger 1982. Die klassische radikale Arbeit ist Glyn/Suttcliffe 1972. Vgl, neuerdings auch Altvater/ Hoffmann/Semmler 1979; Weisskopf 1981; Bowles and Gintis 1982; Bowles 1983.
Dies geschieht in den folgenden drei krisentheoretischen Argumenten, spielt aber auch in verschiedenen interferenztheoretischen Argumenten eine Rolle.

(2) Ebenso wie verteilungspolitischen Argumenten begegnet der Versuch Schwierigkeiten, die Ausdehnung des Staatssektors für ökonomische Stagnation verantwortlich zu machen (vgl. zur Literatur Block 1981). Im internationalen Vergleich besteht kaum eine Korrelation zwischen Niveau oder Wachstumsraten des Staatssektors und des BSP (Castles 1982: 66). Zwar findet sich in den sechziger und frühen siebziger Jahren ein Zusammenhang zwischen relativer Größe des Staatssektors und Stärke der politischen Linken (vgl. Cameron 1978), jedoch gilt dies nicht für Wachstumsraten des Staatssektors in den siebziger Jahren. Zugleich läßt sich in diesem Zeitraum keine überzeugende Korrelation zwischen wirtschaftlicher Leistungsfähigkeit und Größe oder Wachstumsraten des Staatssektors feststellen (vgl. Schmidt 1983: 8f.). Ebensowenig scheinen sozialstaatliche Sicherungssysteme die Arbeitsbereitschaft der Lohnarbeiter gesenkt und ihre Konfliktfähigkeit im Lohnkampf - mit redistributiven Folgen - erhöht zu haben (so aber Vobruba 1979). Denn ein Zusammenhang zwischen höheren Arbeitslosenraten, Sozialausgaben und Lohnquoten kann bislang nicht nachgewiesen werden.

(3) Ein drittes Krisenargument führt die wirtschaftliche Stagnation der siebziger Jahre auf die internationale Einkommensumverteilung durch die Energiepreisschübe der Ölkrisen zurück. Auch wenn einiges für diese These spricht, läßt sich dennoch kaum eine Korrelation zwischen Erdölimportabhängigkeit und Schwere von Wirtschaftskrisen demonstrieren. Japan hätte demnach die größten, die USA die geringsten Wirtschaftsprobleme haben müssen. Tatsächlich verhält es sich jedoch umgekehrt ${ }^{12}$.

Darüber hinaus müssen intensive Auseinandersetzungen um die technischen und ökologischen Folgen der Energieproduktion als Faktor bei der Preissteigerung von Energie berücksichtigt werden. Solche Beobachtungen sind mit Interferenztheorien konsistent, die die soziale Explosivität der „Energiekrisen“" eher als Folge erfolgreichen Wirtschaftswachstums für die soziale und ökologische Infrastruktur auffassen denn als bloße Frage der Einkommensredistribution.

${ }^{12}$ Die Wirkung der Ölschocks auf die OECD-Nationen wird untersucht in Yergin/Hillenbrand 1982. 
(4) Krisentheorien verweisen auf den raschen Strukturwandel der internationalen industriellen Arbeitsteilung als Ursache der anhaltenden Wachstumsstörungen in den entwickelten kapitalistischen Demokratien. Mit dem Aufstieg einer neuen Gruppe industrialisierender Länder (vgl. Anell 1981: 71ff.; Caporaso 1981; Evans 1979; Frieden 1981) sind arbeitsintensive, aber auch technisch und kapitalmäßig anspruchsvollere Produktionssektoren in Hochlohnländern ökonomisch obsolet geworden (Textilien, Stahl, Schiffbau, Konsumelektronik, usw.). Strukturelle Anpassungsprobleme bislang zentraler Industriesektoren mögen tatsächlich erheblich zur Erklärung allgemeiner wirtschaftlicher Trends ebenso wie spezifischer Wachstumsbedingungen in einzelnen Ländern beitragen, besonders wenn auch unterschiedliche staatliche Industriepolitiken berücksichtigt werden.

Allerdings stellt sich die Frage, ob ,wachstumskonforme“ politische Rezepte Strukturkrisen überwinden werden. Interferenztheorien, die eine umfassende materiale Politisierung der Produktion vermuten, wären dann in einer starken Position, wenn sich zeigen ließe, daß die Bedingungen einer wachstumskonformen Krisenüberwindung mit materialen Ansprüchen an die „Sozialverträglichkeit“ neuer Technologien konfligieren.

Reindustrialisierungskonzepte ${ }^{13}$ fordern einen neuen technologischen Innovationsschub und einen Schwerpunkt auf intelligente, energie-, material- und arbeitssparsame, aber wissenschafts- und kapitalintensive Technologien. Die wichtigsten Elemente in der Produktionsfunktion solcher Güter sind mithin Faktoren, die in den sich jüngst industrialisierenden Ländern besonders knapp sind. Zwar ist eine Kompatibilität zwischen Reindustrialisierung und Kriterien einer sozial- und umweltverträglichen Wirtschaftsentwicklung nicht stets ausgeschlossen (vgl. Huber 1982). Aber es sind gerade verschiedene "high technologies“, die bereits bedeutende soziale Konflikte ausgelöst haben, bzw. erwarten lassen. Dabei wäre etwa an Kernenergie, Biotechnologien, fortgeschrittene

\footnotetext{
${ }^{13}$ Angebotsorientierte staatsinterventionistische Strategien der Reindustrialisierung sind auf der politischen Linken (Bowles/Gordon/Weisskopf 1983) und von der neo-merkantilistischen Mitte (Gilpin 1975; Hauff/ Scharpf 1975) vorgeschlagen worden, während marktwirtschaftlich orientierte Konservative einen politisch induzierten Strukturwandel ablehnen: vgl. Institute for Contemporary Studies 1976 mit internationalen Beiträgen.
}

Transportsysteme und neue Kommunikationsmedien zu denken. Ähnlichen Problemen sozialer Akzeptanz mag sich der Vorschlag gegenübersehen, Produktivitätsfortschritte im Bereich persönlicher Dienstleistungen (Erziehung, Gesundheit, Unterhaltung) durch eine „Soziotechnisierung“ unter Einsatz von Mikroelektronik und Informationstechnologie voranzutreiben und damit einen neuen Wachstumsboom auszulösen ${ }^{14}$. Eine dritte Strategie schließlich will der Sättigung von Märkten für massengefertigte Konsumgüter dadurch entgegenwirken, daß weitere Nachfrage durch mehr Produktindividualisierung und Qualitätsverbesserungen ausgelöst wird, die nun aufgrund numerisch kontrollierter Werkzeugmaschinen ökonomisch und technisch praktikabel würden (Sabel 1982: Kap. 5; Piore/Sabel 1985).

Alle Reindustrialisierungsstrategien müßten nicht nur nachweisen, daß ihre Empfehlungen quantitativ erhebliche Wirkungen für Wirtschaftswachstum zeitigen könnten, sondern auch das für Interferenztheorien zentrale Problem ansprechen, inwieweit Motive unter Konsumenten vorhanden sind oder geweckt werden können, einer solchen Strategie Folge zu leisten. Interferenztheorien vermuten, daß eine Technisierung sozialer Diensleistungen auf erheblichen Widerstand stoßen wird. Und die Präferenz für kollektive Güter und nicht-kommodifizierbare Lebenschancen mögen in einem Spannungsverhältnis zu Reindustrialisierungsstrategien stehen, welches auch durch individualisierte Konsumgüter kaum aufgelöst wird.

(5) Die „neuen sozialen Bewegungen“, welche wirtschaftliche Wachstums- und politische Bürokratisierungstrends angreifen, werden von Krisentheorien nicht als eine Ursache für die wirtschaftliche Stagnation der siebziger Jahre und gestiegene politische Konfliktniveaus verstanden, sondern als Folge von Verteilungsproblemen und Wachstumsschwächen. Nach dieser Auffassung ist es ein Mangel an Aufstiegschancen in gesellschaftliche Elitepositionen, welcher hochgebildete Mitglieder der jüngeren Generation zum kollektiven Protest gegen die Organisationsprinzipien des politischen Kapitalismus herausfordert. Solche Akteure kritisieren eine Gesellschaft, die die Elitezirkulation blockiert hat (Bürklin 1983). Allerdings beruht diese These nicht nur auf einer empirisch bislang kaum zu rechtfertigenden Zurechnung von Moti-

14 Vgl. zu dieser Problematik Gershuny 1978 und Gershuny/Miles 1983: Kap. 9. 
ven auf Verhaltensdispositionen ${ }^{15}$, sondern vernachlässigt auch, daß viele Anhänger der ,Wohlstandsgeneration" der heute 30 bis 45 jährigen bereits vor Eintritt der Wirtschaftskrise eine qualitative Kritik des politischen Kapitalismus initiierten und auch heute noch - trotz gelungener Aufwärtsmobilität im vergangenen Jahrzehnt - teilen. Der Zusammenhang zwischen sozialem Status, Bildung und Alter ist komplizierter, als einfache Frustrations-Elitezirkulationsthesen glauben machen, die sich als Erklärung sozialer Bewegungen und sozialer Revolutionen bei Konservativen schon immer großer Beliebtheit erfreuten ${ }^{16}$.

Krisentheorien sind damit insgesamt kaum in der Lage, die Schwächung von Sozial- und Systemintegration und die Erosion des Sozialstaatskompromisses in fortgeschrittenen kapitalistischen Demokratien umfassend zu erklären. Es ist deshalb sinnvoll, Interferenztheorien auf weitere, und zum Teil alternative, Problemerklärungen zu befragen. Wie schon in Schumpeters Marx-Kritik und Kapitalismustheorie (Schumpeter 1975: 42f.; 139) werden Entwicklungsprobleme des Kapitalismus hier nicht aus dem internen Scheitern des Akkumulationsund Verteilungsprozesses erklärt, sondern aus den Folgekosten wirtschaftlichen Wachstums für die selbst nicht kapitalistisch produzierten sozialen, motivationalen und natürlichen Voraussetzungen dieser Produktionsweise. Dieser auch schon in Webers Theorie der Bürokratisierung des modernen Kapitalismus (vgl. Lehnhardt 1980) und in Polanyis (1957) Kritik an der Instabilität selbstregulativer Marktsysteme ausgearbeitete Argumentationslogik liegt die These zugrunde, daß der politische Kapitalismus über keine endogene institutionellen Mechanismen verfüge, selbstdestruktive Interferenzen seines Expansionsprozesses mit seiner Ressourcen- und Motivationsbasis zu antizipieren und entgegenzusteuern ${ }^{17}$. Politik und Staat fallen in diesem Prozeß schon deshalb keine autonome, vorausschauende Rolle $\mathrm{zu}$, weil staatliche Ressourcen vom WachstumsprozeB zehren und

${ }^{15}$ Bürklin (1983) argumentiert, daß nur unter der jüngeren Generation Bildung eine signifikant erklärende Variable für die Unterstützung der wachstumskritischen „Grünen" Partei in der Bundesrepublik ist. Er legt jedoch keine Daten vor, die belegten, daß mangelnde Aufstiegsmöglichkeiten für Gebildete parteipolitische Präferenzen beeinflussen.

${ }^{16}$ Vgl. zur Kritik an Frustrationstheorien sozialer Mobilisierung McCarthy/Zald 1977; Tilly 1978 und Jenkins 1981. konfliktfähig organisierte Interessen sich in politischen Parteien und Interessenverbänden um die Produzentenklassen zentrieren (vgl. Offe 1981; 1983). Erst Probleme des „Marktversagens“ und der Intensivierung sozialer Konflikte setzen Rückkoppelungsprozesse in Gang, die auf diese Problemlage reagieren. Interferenztheorie verfügen über mindestens fünf Argumente, die eine materiale Politisierung der Produktion mit ihren ökonomischen Stagnations- und politischen Konflikterscheinungen erklären sollen.

(1) Eine heute weitverbreitete naturalistische, unsoziologische Theorie behauptet, daß der moderne Kapitalismus die Naturbasis gesellschaftlicher Produktion zerstöre und bereits heute physische Wachstumsgrenzen erreicht habe, die direkt oder indirekt auf thermodynamische Gesetzmäßigkeiten zurückgeführt werden könnten. ${ }^{18}$. Diese These beruht auf starken Annahmen über die Unmöglichkeit weiteren technischen Fortschritts und läßt außer Acht, daß die Ökologieproblematik unentwirrbar mit sozialen und kulturellen Evaluationen natürlicher Lebensbedingungen verknüpft ist.

In einer soziologischen Fassung des Ressourcenarguments wird die Politisierung der Naturnutzung auf mindestens zwei gesellschaftliche Ursachen zurückgeführt. Erstens werden Naturressourcen durch technologische Fortschritte immer mehr gesellschaftlichen Wahlentscheidungen über ihre Nutzung, Erhaltung oder Zerstörung zugänglich gemacht (vgl. Ruggie 1975: 560). Dabei vergrößert sich jedoch die Diskrepanz zwischen Eingriffskapazitäten und kognitiven Fähigkeiten zur Evaluation von Eingriffsfolgen. Unsicherheit über die Folgen menschlicher Natureingriffe bilden das größte Entscheidungsproblem (Elster 1979). Technische Optionen und unsichere Evaluationsbedingungen fördern gleichermaßen eine Politisierung der Naturnutzung. Zweitens verwendet der politische Kapitalismus Naturressourcen immer ausschließlicher für Zwecke der Güterproduktion, ohne Präferenzen für andere Nutzungsbezüge (Natur als soziales Konsum- und Reproduktionsfeld) in dem Maße in Rechnung zu stellen, wie es bislang

17 Unter modernen Interferenztheorien finden sich insbesondere die Arbeiten von Habermas $(1973 ; 1981)$ und Touraine (1977a), deren soziologisches Abstraktionsniveau zumeist höher liegt als in den Thesen dieses Essays.

18 Prominente Vertreter dieser Position sind Commoner 1971; Georgescu-Roegen 1975; Ophuls 1977; Rifkin 1980. 
kulturellen Selbstverständlichkeiten selbst in modernen Gesellschaften entsprach.

Die Politisierung der Naturnutzung wird durch den Sachverhalt verstärkt, daß sich Entscheidungen zwischen alternativen produktiven oder konsumtiven Nutzungsweisen nicht nach Maßgabe marktwirtschaftlicher Spielregeln treffen lassen, die Allokationsprobleme segmentieren und entpolitisieren könnten. Bei vielen kollektiven Naturgütern lassen sich Eigentumsrechte nicht privatwirtschaftlich definieren und deshalb Kosten und Nutzen alternativer Naturverwendung nicht umstandslos auf einzelne „Eigentümer“ zurechnen. Darüber hinaus liegen keine praktikablen marktwirtschaftlichen Verfahren vor, die aggregierten Gesamtkosten alternativer Nutzungsformen kollektiver Güter und Externalitäten monetär zu bestimmen und die Kostenträger angemessen zu kompensieren ${ }^{19}$. Sofern Naturressourcen schließlich nicht-erneuerbar sind, scheitert eine nach Maßgabe von Märkten effiziente intertemporale Allokation an kritischen Informationsmängeln (über die GesamtgröBe der wirtschaftlich gewinnbaren Vorräte einer Ressource; zukünftige Extraktionstechnologien; Kosten und Verfügbarkeit von Substituten; Nutzungspräferenzen/Nachfrage zukünftiger Generationen; Diskontsätze bei der Bewertung zukünftigen Nettonutzens der Ressourcenextraktion usw. $)^{20}$.

Eine wachstumshemmende Wirkung materialer Politisierung der Naturnutzung ist im vergangenen Jahrzehnt unter Stichworten wie „zuviel Regulation" und "Politisierung des Eigentumsbegriffs" von neoklassischen Ökonomen beklagt worden. Sie bewirkte eine Verlängerung der „Produktionsumwege" von Rohstoffen zu Endprodukten, wobei Umweltschutz und sparsame Ressourcennutzung die Kapitalintensität der Produktion erhöhten, wie sich im Energiebereich und anderen Industrien mit hoher Umweltbeeinträchtigung nachweisen läßt. Allerdings ist angesichts der Unsicherheit über zukünftigen technischen Fortschritt die Möglichkeit nicht umstandslos von der Hand zu weisen,

${ }^{19}$ Mit diesem Problem hat sich die Umweltökonomie (vgl. als Überblick: Downing 1984) bislang mit wenig befriedigendem Ergebnis auseinandergesetzt (vgl. kritisch Ophuls 1977: Kap. 5).

${ }^{20}$ Zur Ökonomik erschöpflicher Ressourcen vergleiche aus neoklassischer Sicht Solow 1974 und Siebert (Hg.) 1980; zur ökologischen Kritik Georgescu-Roegen 1975, Schefold 1977 und Meixner 1980. daß in der Zukunft ,intelligente“ Technologien Ansprüche von Umweltschutz und ökonomischer Effizienz wechselseitig optimieren könnten. Träfe dies zu, dürften umweltpolitische Argumente allein kaum ausreichen, eine dauerhafte materiale Politisierung der Produktion zu erwarten.

(2) Eine zweite Ursache der materialen Politisierung der Produktion ergibt sich aus den Bedingungen von Gewinnung, Verteilung und Nutzung von Information, die in "post-industriellen“ Gesellschaften eine zentrale Stellung bei der Steuerung des gesellschaftlichen Prozesses gewinnen (Bell 1973; Touraine 1971). Theorien effizienter Marktallokation haben Schwierigkeiten, die Rolle der Information im Marktsystem zu bestimmen (vgl. kritisch Parsons/Smelser 1956: 11f.; Attali 1976: 124). Trotz großer Anstrengungen in der ökonomischen Analyse von Informationskosten und von informationeller Unsicherheit (vgl. Hirshleifer/Riley 1979), bleibt das Paradox unaufgelöst, daß Information zum einen wie jede andere Ware produziert und gehandelt werden kann, zum anderen aber auch - im Sinne des Verfügens über Preis-, Qualitäts-, Bedienungs-, Funktions- und Wirkungsinformation - Voraussetzung effizienten marktwirtschaftlichen Äquivalententauschs ist (Grossman/Stiglitz 1980). Vollständige Information, d. h. auch: die kostenlose Dissemination von Information, bleibt eine Bedingung effizienter Märkte (Posner 1981). Informationsasymmetrien sind eine zentrale Ursache, warum Märkte von interner Organisation/Politik als Transaktionssystem abgelöst werden ${ }^{21}$.

Durch die zunehmende Verwissenschaftlichung von Produktion und Produkten wird Zugang zu Information gerade für Konsumenten und Kleinproduzenten zu einem kritischen politischen Thema. Ökonomische Effizienzargumente bilden dabei nur einen Argumentationsstrang, um politische „Regulation“ zu rechtfertigen (cf. Ordover/Weiss 1981; Beales/Croswell/Salop 1981). Denn jenseits ökonomischer Rationalitätskriterien entscheidet die Allokation von Information zuallererst darüber, wer überhaupt den Status eines kompetenten Marktteilnehmers und Staatsbürgers erlangen kann und wer durch exklusives Verfügen über Information politische und wirtschaftliche Prozesse kontrolliert. Der Kampf um Information betrifft damit Ökonomie und Politik zugleich. In der wirtschaftlichen Sphäre geht es etwa um den Eigen-

${ }^{21}$ Vgl. Williamson 1975 zum Problem der ,information impactedness". 
tumsschutz im Patentrecht, dem gegenüber die Öffentlichkeit ein „Recht auf Information“ - etwa über Risikopotentiale neuer Technologien - geltend macht. Zugleich sind professionelle Wissensmonopole (in Medizin, Recht, Technik) zunehmend Zielscheibe öffentlicher Kritik geworden, weil ihr Bedeutungsgewinn im gesellschaftlichen Prozeß asymmetrische Kommunikationssituationen zementiert.

Schließlich äußert sich die Politisierung des Informationswesens in vielfältigen Auseinandersetzungen um die Anlage privater und öffentlicher Datenbanken, den öffentlichen Zugang zu solchen Informationssystemen (Stichwort „Freedom of Information Act") und den Schutz der Privatsphäre vor dem Zugriff von Datensammlern in Wirtschaft und politischer Bürokratie.

Es dürfte zwar kaum möglich sein, die ökonomische Stagnation der siebziger Jahre auf die Politisierung des Informationswesens zurückzuführen. Jedoch stellt diese einen wichtigen Ausgangspunkt für die Mobilisierung sozialer Bewegungen dar. Darüber hinaus läßt sich vorläufig nur darüber mutmaßen, welche Folgen der Wandel des Informationswesens für die gegenwärtigen politischökonomischen Organisationsformen haben mag. In einem Extremfall mag die Hierarchisierung und Spezialisierung von Information Demokratie und Marktfreiheit fragwürdig machen. Nach einer diametral entgegengesetzten These (die von Kochen/ Deutsch 1980: Kap. 8 zumindest implizit nahegelegt wird) drängen neue Informationstechnologien und ihre Regelungsstrukturen auf eine Dezentralisierung von Gesellschaft und den Verlust exklusiven Verfügens über Information, womit sowohl die privatwirtschaftliche als auch die bürokratische Kontrolle von Information zu Fall käme.

(3) Eine dritte Ursache der materialen Politisierung und Produktion bezieht sich auf die Allokation angebotsinelastischer positionaler Güter (Hirsch 1976). Bei positionalen Gütern bleibt die Gratifikationsfähigkeit des Gutes an soziale Knappheit, d. h. die Limitierung von Produktion und Konsum des Gutes gebunden. Exzessiver Genuß positionaler Güter führt zum Wertverlust der Güter durch Überfüllung, Verstopfung, Verlust der Nutzungsexklusivität. Die Verteilungslogik positionaler Güter ist deshalb ein Nullsummen- oder sogar Negativsummenspiel absoluter Knappheit. Zwar liefert der politische Kapitalismus in Positivsummenspielen immer mehr angebotselastische Güter. Da jedoch positionale Güter anscheinend größere Befriedigung verschaffen als angebotsela- stische (Scitovsky 1975) und mit steigenden Einkommen vermehrt nachgefragt werden, ohne eine Angebotsexpansion hervorzurufen, bildet sich hier potentieller politischer Konfliktstoff.

Die Preise absolut knapper Güter eskalieren nicht nur über die allgemeine wirtschaftliche Inflationsrate hinaus (z. B. in Bodenspekulation) und enttäuschen Konsumenten, die sich von höheren Geldeinkommen Zugriff auf mehr positionale Güter erwarteten. Sondern vermehrte Geldeinkommen selbst beruhen auf einer Ausdehnung der Produktion angebotselastischer Güter und Leistungen, die die Tendenz haben, positionale Güter zu verdrängen. Auseinandersetzungen um die räumliche Organisation moderner Gesellschaften (Standortplanung für Industrieanlagen und Verkehrstrassen; Stadt- und Regionalplanung) oder der Mobilitätsdruck auf Arbeitskräfte liefern Beispiele für Null- und Negativsummenspiele um positionale Güter.

Politische Resistenz gegen die Subordination positionaler Güter unter wirtschaftliche Effizienzkriterien erhöht private Investitionsrisiken und dämpft Investitionsbereitschaft. Konsumentenenttäuschung über den Nutzwert angebotselastischer Güter und die Unerreichbarkeit positionaler Güter trägt zur Stagnation der Nachfrage nach ersteren bei. Darüber hinaus wirft die Auseinandersetzung über positionale Güter schließlich auch Zweifel an der Legitimität eines Gesellschaftssystems auf, welches Positivsummenspiele zu einer zentralen Legitimationsformel gemacht hat.

(4) Eine vierte Ursache materialer Politisierung der Produktion ergibt sich aus dem zunehmenden Unbehagen von Bevölkerungsgruppen mit marktwirtschaftlich oder politisch-bürokratisch produzierten persönlichen Dienstleistungen. Darunter seien solche Aktivitäten verstanden, die unmittelbar zur symbolischen und physisch-psychischen Identitätsbildung von sozialen Akteuren beitragen (Erziehung/Bildung; Gesundheit/Medizin; Kultur; etc.). Sozialdienste widersetzen sich erstens dem marktwirtschaftlichen Äquivalententausch, weil angesichts unsicherer und komplexer Produktionstechnologien und Interdependenz zwischen Leistungsträgern und Klienten kein dem Markttausch angemessener Vertrag über die wechselseitigen Verpflichtungen der Tauschparteien ausformuliert werden kann. Kommodifizierte Sozialleistungen sind deshalb oft für die Klienten enttäuschend, wenn sie ihre privaten, nicht vertraglich spezifizierten Erwartungen über die Ergebnisse der Transaktion nicht erfüllt sehen. Hohe marktwirtschaftliche 
Transaktionskosten führen zur Organisation von Dienstleistungen ohne Äquivalententausch, etwa in Firmenabteilungen oder politischen Bürokratien $^{22}$. Im Fall von persönlichen Dienstleistungen mag die bürokratische Mediatisierung der Tauschrelation zwischen Dienstleister und Klient das Frustrationspotential jedoch nur noch erhöhen, da Leistungserwartungen nicht durch Marktpreise gezügelt werden (vgl. Hirschman 1981: 39ff.).

Zweitens entgeht sowohl marktförmigen als auch bürokratischen persönlichen Dienstleistungen eine kommunikative Qualität, welche Leistungsanbieter und Klient nicht nur instrumentell als wechselseitige Mittel zur individuellen Bedürfnisbefriedigung miteinander verbindet, sondern die soziale Beziehung als Selbstzweck konstituiert, in der gemeinsame Situationsdefinitionen und Normen entwickelt, bekräftigt und bewahrt werden. Lebensweltliche Aktivitäten innerhalb eines symbolisch konstituierten sozialen Universums erweisen sich deshalb gegenüber marktförmiger oder bürokratisch-rechtlicher Organisation als resistent ${ }^{23}$. Gegen den bürokratisierten Sozialstaat erheben Klienten sozialer Dienstleistungen Forderungen nach Autonomie, Solidarität und neuen primären sozialen „Ligaturen“ (Dahrendorf 1979) ${ }^{24}$. Da der Gratifikationswert primärer Sozialkontakte höher ist als derjenige materieller Güter und bürokratischer Dienstleistungen (vgl. Scitovsky 1975; Leiss 1976; Lane 1978), haben solche Konflikte deshalb nicht nur tendenziell wirtschaftlich entstabilisierende, sondern auch potentiell delegitimierende Folgen für die Institutionen des politischen Kapitalismus.

(5) Konsumentenenttäuschung und Wahrnehmung kontra-intuitiver Folgen der Wachstumswirtschaft schwächen schließlich auch die für Konkurrenzökonomien konstitutive Produzentenethik gerade

${ }^{22}$ Prohibitive Transaktionskosten, sind ein zentrales Argument für die Entstehung von Firmen anstelle marktförmiger Koordination. Vgl. Williamson 1975.

${ }^{23}$ Habermas 1981: 538ff. Allerdings bleibt Habermas' These problematisch, daß traditionelle Normbestände und lebensweltliche Selbstinterpretationen heute in einer Weise erodiert sind, daß nur noch eine postkonventionelle Moral, die einer prozeduralen Diskursethik aufruht, soziale Normen begründen kann

${ }^{24}$ Trade-offs zwischen Freiheit, Gleichheit und Sicherheit in der Sozialpolitik werden bei Heclo (1981) diskutiert. Zum Programm einer Entformalisierung des Dienstleistungssektors vgl. Binswanger et al. 1979. bei ökonomisch saturierten und/oder kognitiv und normativ hochsensibilisierten Bevölkerungsgruppen, die „qualitative Lebenschancen“ warenförmigen und bürokratischen Leistungen vorziehen. Wirtschaftliche Leistungsmotivationen lassen in dem Maße nach, in welchem die Steigerung von Wirtschaftsergebnis und von sozialen Lebenschancen nicht mehr als gleichsinniger Prozeß wahrgenommen werden. Es mag jedoch verfehlt sein, dieses Phänomen als Revolution steigender Ansprüche (Crozier et al. 1975) oder „Wertwandel“" (Inglehart 1977) zu identifizieren. Neben empirisch-methodologischen und begrifflichen Problemen der Wertwandelsthese (vgl. zusammenfassend Müller-Rommel 1983) ist es auch theoretisch unplausibel, von einem „Ansteigen“ gesellschaftlicher Anspruchsniveaus zu sprechen ${ }^{25}$. Angesichts zunehmender Bildungskompetenz läßt sich allenfalls ein Anstieg kognitiver Kapazitäten feststellen, eine Gefährdung oder einen Verlust qualitativer Lebenschancen wahrzunehmen. Allerdings wurzeln die sich aus solchen Prozessen ergebenden Konflikte und Proteste durchaus in ,alten" bürgerlichen und vorbürgerlichen Werten von Autonomie, sozialer Bedürfnisbefriedigung und Solidarität. Untersuchungen zum Wertwandel mögen deshalb eher eine Verschiebung der handlungsleitenden Relevanz (salience) von Werten im gegenwärtigen Kontext sozialen Wandels beobachten als eine Transformation der Wertbasis selbst. Nicht „neue Werte", sondern die expansive Logik eines politisch-ökonomischen Systems, welches bislang über keine hinreichenden selbstregulativen Mechanismen verfügt, der Unterhöhlung seiner Ressourcen- und Motivationsbasis vorzubeugen, erklärt die Vielzahl von Interessengruppen und sozialen Bewegungen, die sich in modernen Demokratien um qualitative Lebenschancen bemühen. Zugleich muß Gesellschaftstheorie bei der Untersuchung sozialer Akteure kognitiven Wahrnehmungsprozessen große Bedeutung beimessen und damit die Hegemonie normativer Erklärungen aufheben ${ }^{26}$.

${ }^{25}$ Allenfalls läßt sich mit Bell (1976) ein zunehmender, historisch tiefverwurzelter und auch funktional im kapitalistischen Reproduktionskreislauf begründeter Dualismus von asketischer Produktions- und hedonistischer Konsumethik vermuten.

${ }^{26} \mathrm{Vgl}$. allgemein Warner 1978. Politik ist wiederholt als kognitiver Lernprozeß aufgefaßt worden (Heclo 1974: 304ff.; Deutsch 1981: 338), ohne jedoch Lernschranken systematisch zu spezifizieren (vgl. kritisch: Lindberg 1976: 254-261). 
Insgesamt nehmen soziologische Interferenztheorien immer wieder zwei empirische Referenzen in Anspruch, um die These eines "Periodenbruchs" sozialer Integrationsprozesse in kapitalistischen Demokratien zu begründen. Zum einen haben sich Auseinandersetzungen um Phänomene des „Marktversagens" in einer Weise intensiviert, daß immer mehr Forderungen nach einer „Regulation“ und demokratischen Kontrolle des politischen Kapitalismus auftreten. Debatten um Ausmaß und Zweck politischer Regulation sind deshalb neben Fragen der Einkommensverteilung ins Zentrum der politischen Auseinandersetzung gerückt. War Wirtschaftsregulation bis dahin vornehmlich ein kooperativer Koordinationsprozeß zwischen Staat und Industrie nach Maßgabe wirtschaftlicher Erfolgskriterien, so ist Regulation nun oft ein konfliktreicher Prozeß, in dem die Rationalitätskriterien politischer Steuerung selbst umstritten sind. Zum anderen haben soziale Bewegungen dabei jedoch nicht nur die Inhalte regulativer Politiken herausgefordert, sondern auch die institutionellen Entscheidungsregeln der Politik selbst. Stichworte sind hierbei die Kartellisierung des politischen Prozesses durch bürokratisierte Parteien und die Verbändeherrschaft eines auf ökonomisch konfliktfähige Produzenteninteressen eingeschränkten Neokorporatismus (vgl. Berger 1979; Offe 1980). Der politische Kapitalismus ist mithin sowohl hinsichtlich seiner ökonomischen als auch seiner politischen Strukturprinzipien ins Kreuzfeuer der Kritik geraten.

Allerdings ist die „Verstopfung“ des politischen Prozesses durch eine Proliferation neuer Gruppen und Ansprüche im politischen ProzeB, die etwa von prominenten Vertretern der ,Unregierbarkeits"these zeitgenössischer Demokratien (wie Crozier et al. 1975; Heclo 1978 und Olson 1982) für politisch ökonomische Integrationsprobleme verantwortlich gemacht wird, selbst nur eine vordergründige Erklärung für Wachstumsprobleme. Erst die Argumente von Interferenztheorien zeigen, warum in den vergangenen Dekaden eine Explosion politischer Konflikte stattgefunden hat. Zwar ist die Forderung nach qualitativen sozialen Lebenschancen, jenseits von Märkten und politischen Bürokratien, keineswegs völlig neu, sondern historisch wohlvertraut aus defensiven Bewegungen gegen die Frühindustrialisierung (vgl. Thomp-

${ }^{27}$ Zur Problematik „regulativer" Wirtschaftspolitiken siehe Sabatier 1975; Mitnick 1980; Wilson 1980 und zuletzt Lehner et al. 1984. son 1966), gegen die Verdrängung selbständiger Kleinunternehmer (in rechtsradikalen Bewegungen) und gegen den Statusverlust des Bildungsbürgertums und der unabhängigen Intelligenz in der Industrialisierung (etwa in Anarchismus, Boheme und Gegenkultur). Dennoch unterscheiden sich heutige soziale Bewegungen, die eine materiale Politisierung der Produktion fordern, von solchen Erscheinungen quantitativ durch die Breite sozialer Mobilisierung und qualitativ durch die strukturellen Kontextbedingungen ihres Protests sowie die Lokalisierung der zentralen Akteure im Gesellschaftssystem: Sie befinden sich weniger an der Peripherie der gesellschaftlichen Dynamik (wie die defensiven sozialen Proteste) als in ihrem Zentrum (etwa als Beschäftigte im Sektor sozialer Dienstleistungen). Und sie verfügen über kognitive und organisatorische Ressourcen, die kaum mit der Mobilisierung defensiven Protests gegen die Industrialisierung vergleichbar sind.

All dies heißt nicht, daß ein historischer „Periodenbruch" zugleich soziale Revolution herbeiführen wird. In der historischen Entwicklung haben heutige kapitalistische Demokratien neue Konfliktachsen oft durch allmählichen Strukturwandel assimiliert. Interferenztheorien sind nicht Zusammenbruchstheorien, auch wenn die zuweilen apokalyptische Rhetorik einzelner Akteure sozialer Bewegungen dies nahelegen mögen. Vielmehr werden die politisch-ökonomischen Folgen der materialen Politisierung der Produktion wesentlich durch institutionelle Chancenstrukturen im politischen System beeinflußt. Solche Konsequenzen dürften dabei umso inkrementeller eintreten, je größer die Permeabilität politischer Verfahren für die Beteiligung und Repräsentation neuer Ansprüche ausfällt und je größer die Responsivität politischer Institutionen und Eliten im Prozeß der Politikformierung und -implementation gegenüber solchen Forderungen ist. Zwischen Assimilation und Disruption moderner kapitalistischer Demokratien eröffnet sich damit ein breites Feld von Entwicklungsmöglichkeiten.

\section{Konsequenzen und Forschungsperspektiven}

Aus dem hier vorgestellten vorläufigen Theorierahmen lassen sich eine Reihe empirisch prüfbarer Hypothesen ableiten. Solche Forschungsfragen legen auch bestimmte Untersuchungsmethoden und Studienobjekte nahe. 
Meine erste These lautet, da $\beta$ in der Sequenztheorie eine materiale Politisierung der Produktion erheblich von der institutionellen Bearbeitung vorhergehender Entwicklungsherausforderungen abhängt. Konkret bedeutet dies, daß eine breite und umfassende Institutionalisierung des Sozialstaates eine wesentliche Bedingung für eine tiefgreifende materiale Politisierung der Produktion darstellt. Länder, in denen eine solche Institutionalisierung des Sozialstaates (noch) nicht eingetreten ist, wie etwa die USA (vgl. Kudrle/Marmor 1981) oder auch Japan, erfahren eine Politisierung der Produktion vorläufig weniger konsistent als etwa die sozialstaatlichen Demokratien Nordeuropas. Selbstverständlich ist dies nicht die einzige Bedingung, die unterschiedliche Entwicklungspfade der materialen Politisierung der Produktion, etwa durch die Mobilisierung sozialer Bewegungen, zum heutigen Zeitpunkt erklärt (vgl. Kitschelt 1983b und 1984b).

Meine zweite These wendet sich gegen krisentheoretische Erklärungen des Periodenbruchs der siebziger Jahre. Während diese voraussagen, daß eine Korrektur der Verteilungsverhältnisse in modernen politischen Ökonomien die materiale Politisierung der Produktion beseitigt, vermuten Interferenztheorien keinen solchen Zusammenhang. Mehr noch, Interferenztheorien erwarten eine Verschärfung von solchen Konflikten, sofern der politische Kapitalismus zu den aus der Vergangenheit bekannten Wachstumsrhythmen zurückkehrte. Die neuen sozialen Konflikte sind nicht primär eine Antwort auf wirtschaftliche Stagnation, sondern eher eine Folge wirtschaftlichen Wachstums und eine Krisenursache.

Meine dritte These vermutet als Implikation der materialen Politisierung der Produktion die dauerhafte Bildung einer neuen Achse sozialer Mobilisation und neue Koalitionen sozialer Gruppen. Diese Entwicklung deutet sich bereits durch die weithin beobachtbare Neuorientierung westeuropäischer Parteiensysteme seit den siebziger Jahren an, in denen sich entweder neue Parteien (wie Grüne und Ökologen in Frankreich, Belgien, der Bundesrepublik usw.) bilden und/oder traditionelle liberale bis sozialistische Parteien sich auf neue gesellschaftspolitische Aggregationsmuster und Koalitionen einstellen. Während Lipset/Rokkan (1967) in den sechziger Jahren noch eine eigentümliche Stabilität der Struktur von Parteiensystemen über einen Zeitraum von vier Jahrzehnten beobachten konnten, ist diese Stabilität nun aufgebrochen worden. Mit dem Auftreten neuer Konfliktachsen ha- ben sich auch die ideologischen Fronten repolarisiert (Pizzorno 1981: 272).

Meine vierte These betrifft unmittelbar heute geläufige gesellschaftspolitische Programme. Sofern das vorgeschlagene Entwicklungsmodell und die Interferenztheorie zutreffen, haben politische Strategien wenig Aussicht auf Erfolg, die den Periodenbruch der siebziger Jahre mit Mittel angehen, welche zur Bearbeitung vorhergehender Integrationsprobleme vielleicht sinnvoll gewesen wären. Sie garantieren weder eine dauerhafte Restauration der Wachstumswirtschaft noch eine Zerstreuung der Protestbewegungen, die sich auf die materiale Politisierung der Produktion richten. Sowohl Neoliberalismus als auch Neokeynesianismus sind deshalb kaum aussichtsreich: Die bereits absehbaren Grenzen neoliberaler (Großbritannien, USA) und neokeynesianischer Experimente (Frankreich, Schweden) lassen zwar noch keine schlüssige Beurteilung zu, legen diese Einschätzung jedoch nahe. Insgesamt vielversprechender erscheinen die miteinander weitgehend inkompatiblen Strategien einer neo-merkantilistischen industriellen Modernisierung (Japan) oder einer bisher unversuchten Neutralisierung des Wirtschaftswachstums. Allerdings kann die erstere Strategie nur erfolgreich sein, wenn die sozialen Bewegungen, welche den Wachstumsprozeß kritisieren, eingedämmt und wirkungslos gemacht werden können. Und eine Neutralisierung des Wachstums beruft sich auf vorläufig noch vage Vorstellungen einer Begrenzung marktwirtschaftlicher Allokationsformen, eine „Autozentrierung“ regionaler Wirtschaftsgebiete durch Ablösung vom Weltmarkt und eine Neubildung demokratischer politischer Entscheidungsformen ${ }^{28}$, ohne jedoch die dabei sich stellenden Macht- und Herrschaftsfragen gesellschaftlichen Wandels eingehend zu reflektieren. Da zur Zeit nicht abzusehen ist, welche Gesellschaftskonzeptionen machbar und durchsetzungsfähig sind, bleibt weiterhin eine große Variabilität der Wirtschafts- und Sozialpolitiken kapitalistischer Demokratien zu erwarten.

Die vielleicht aussichtsreichste Strategie für die Analyse konkurrierender Theorien über die Des-

${ }^{28}$ Vgl. aus der Fülle von Entwürfen und utopischen Spekulationen über eine Neutralisierung des Wirtschaftswachstums: Attali 1978; Daly 1977; Johnson 1979; Rosier/Dockès 1983; Huber 1980, 1982; Strasser/ Traube 1981. Zur normativen politischen Theorie in „ökologistischen“" Gesellschaftsvisionen siehe Kitschelt 1984a: Kap. 5. 
integration des Sozialstaatskompromisses in den siebziger Jahren ist die vergleichende Politik- und Institutionenforschung. Dabei müßten jedoch in mindestens zwei Hinsichten neue Schwerpunkte gesetzt werden. Die vergleichende Politikforschung hat sich bislang vorwiegend auf wirtschaftspolitische Verteilungsfragen konzentriert, jedoch genau jene Strukturpolitiken zumeist vernachlässigt, die in der materialen Politisierung der Produktion den meisten Konfliktstoff liefern. Dies gilt etwa für Verkehrs- und Raumplanung, Energieund Umweltpolitik, Technologie- und Informationsentwicklung, aber auch qualitative Aspekte der Institutionalisierung und Steuerung sozialer Dienstleistungen. Des weiteren müßten solche Untersuchungen mit Hilfe eines komplexeren begrifflichen und theoretischen Instrumentariums angegangen werden als viele der vorliegenden vergleichenden Politikanalysen, die sich zumeist auf „konjunkturelle“ politische und ökonomische Determinanten der Politikformierung beschränken, ohne die „strukturellen“ Designs politischer Entscheidungsprozesse genauer zu berücksichtigen.

Ein anderer Schwerpunkt besteht in der empirisch vergleichenden Untersuchung der sozialen Akteure, die sich in sozialen Bewegungen um die materiale Politisierung der Produktion bemüht haben. Bislang liegen kaum vergleichende Arbeiten zu diesem Thema vor (vgl. als Anfang: Brandt 1985). Die Theorie sozialer Bewegungen ist zudem fast ausschließlich ein Feld der angloamerikanischen und französischen Soziologie und Politikwissenschaft geblieben. Jedoch können theoriegeleitete Untersuchungen über Entstehungsbedingungen, Organisationsformen und Strategien sozialer Bewegungen Aufschluß über die Dauerhaftigkeit neuer gesellschaftspolitischer Forderungen geben.

Es gilt also, aus den in den vergangenen Jahren sich abzeichnenden Verschiebungen und Neubildungen gesellschaftlicher Konfliktlinien und Diskussionsthemen auch für Untersuchungsprioritäten der Sozialwissenschaften Konsequenzen zu ziehen. Allerdings heißt dies nicht, daß die Methodik und argumentative Selbstdisziplin erfahrungswissenschaftlicher Studien zugunsten utopischen Wunschdenkens und „soziologischer Intervention“ (im Sinne Touraines, 1978) aufgegeben werden müssen.

\section{Literatur}

Altvater, E./J. Hoffmann/W. Semmler, 1979: Vom Wirtschaftswunder zur Wirtschaftskrise. Berlin: Olle und Wolter.
Anderson, P., 1974: Lineages of the Absolutist State. London: New Left Books.

Anell, L., 1981: Recession. The Western Economies and the Changing World Order. New York: St. Martin's Press.

Appleby, J., 1978: „Modernization Theory and the Formation of Modern Social Theories in England and America". Comparative Studies in Society and History, 20: 259-285.

Alford, R. and R. Friedland, 1974: „Nations, Parties, and Participation. A Critique of Political Sociology“. Theory and Society, 1: 307-328.

Attali, J., 1976: La parole et l'outil. Paris (zweite Auflage): Presses Universitaires de France.

Attali, J., 1978: La nouvelle économie française. Paris: Flammarion.

Autorengruppe, 1981: „Zyklische Rhythmen und sakulare Trends der kapitalistischen Weltwirtschaft: Einige Prämissen, Hypothesen und Fragen“. in: F. Fröbel/J. Heinrichs/O. Kreye ( $\mathrm{Hg}$.), Krisen in der kapitalistischen Weltökonomie. Reinbek: Rowohlt, S., 101-119.

Beales, H./R. Crosswell/S. Salop, 1981: „Information Remedies for Consumer Protection", American Economic Review, Papers and Proceedings, 71: 299-304.

Bell, D., 1973: The Coming of Post-Industrial Society. A Venture in Social Forecasting. New York: Basic Books.

Bell, D., 1976: The Cultural Contradictions of Capitalism. New York: Basic Books.

Bendix, R., 1977: Nation-Building and Citizenship. Enlarged edition. Berkeley: University of California Press.

Berger, S., 1979: „Politics and Anti-Politics in Western Europe in the Seventies“. Daedalus, 108: 27-50.

Bergmann, J./G. Brandt/K. Korber/O. Mohl/C. Offe, 1968: „Herrschaft, Klassenverhältnis und Schichtung“. S. 67-87 in: T. W. Adorno (Hg.), Spätkapitalismus oder Industriegesellschaft? Stuttgart: Enke.

Binder, L., 1971: „Crises of Political Development“. S. 3-72 in: L. Binder (ed.), Crises and Sequences in Political Development. Princeton, N. J.: Princeton University Press.

Binswanger, H./W. Geissberger/T. Ginsburg, 1979: Wege aus der Wohlstandsfalle. Frankfurt: Fischer.

Block, F., 1981: „The Fiscal Crisis of the State“. Annual Review of Sociology, 7: 1-27.

Bowles, S., 1983: „The Post-Keynesian Capital-Labor Stalemate“. Socialist Review, 12: 45-72.

Bowles, S./D. M. Gordon/T. E. Weisskopf, 1983: Beyond the Wasteland. New York: Anchor/Doubleday.

Bowles, S./H. Gintis, 1982: „The Crisis of Liberal Democratic Capitalism: The Case of the United States". Politics and Society, 11: 51-93.

Brandt, G., 1972: „Industrialisierung, Modernisierung, gesellschaftliche Entwicklung. Anmerkungen zum gegenwärtigen Stand gesamtgesellschaftlicher Analysen“. Zeitschrift für Soziologie, 1: 5-14.

Brand, K. W./D. Büsser/D. Rucht, 1983: Aufbruch in eine neue Gesellschaft. Frankfurt/Main: Campus. 
Brand, K. W. (Hg.), 1985: Neue Soziale Bewegungen in Westeuropa und in den USA. Frankfurt/Main: Campus.

Bürklin, W. P., 1983: Ansatzpunkte einer sozialstrukturellen Verankerung der neuen sozialen Bewegungen. Fachtagung der Deutschen Vereinigung für Politische Wissenschaft, 11.-13. 10.1983, Ms.

Cameron, D., 1978: „The Expansion of the Public Economy: A Comparative Analysis“. American Political Science Review, 78: 1243-1262.

Cameron, D., 1982: Social Democracy, Corporatism, and Labor Quiescence. Ms. Conference on Representation and the State. Stanford University.

Caporaso, J., 1981: „Industrialization in the Periphery: The Evolving Global Division of Labor". pp. 140-171 in: W. L. Hollist and J. N. Rosenau (eds.), World System Structure. Continuity and Change. Beverly Hills: Sage.

Castles, F. G., 1982: „The Impact of Parties on Public Expenditure“. pp. 21-96 in: F. G. Castles (ed.), The Impact of Parties. Politics and Policies in Demoratic Capitalist States. Beverly Hills: Sage.

Castles, F. G., 1978: The Social Democratic Image of Society. London: Routledge and Kegan Paul.

Coleman, J. S., 1971: „The Development Syndrome: Differentiation - Equality - Capacity“. pp. 73-100 in: L. Binder et al., Crises and Sequences in Political Development. Princeton, N. J.: Princeton University Press.

Commoner, B., 1971: The Closing Circle. New York: Knopf.

Crozier, M./S. P. Huntington/J. Watanuki, 1975: The Crisis of Democracy. New York: New York University Press.

Dahrendorf, R., 1979: Lebenschancen. Frankfurt/Main: Suhrkamp.

Daly, H., 1977: Steady State Economics. San Francisco: Freeman.

Deutsch, K. W., 1981: „The Crisis of the State“. Government and Opposition. 16: 331-343.

Dobb, M., 1946: Studies in the Development of Capitalism. London. zit. nach revidierter Ausgabe New York: International Publishers.

Downing, P. B., 1984: Environmental Economics and Policy. Boston: Little, Brown.

Elsenhans, H., 1983: „Rising Mass Incomes as a Condition of Capitalist Growth: Implications for the World Economy“. International Organization, 37: 1-39.

Elster, J., 1979: „Risk, Uncertainty, and Nuclear Power". Social Science Information, 18: 371-400.

Emminger, O., 1982: „West Germany - Europe's Driving Force?" pp. 21-45 in: R. Dahrendorf (ed.), Europe's Economy in Crisis. London: Weidenfeld and Nicolson.

Evans, P., 1979: Dependent Development. Princeton, N. J.: Princeton University Press.

Flora, P., 1981: „Stein Rokkans Makro-Modell der politischen Entwicklung Europas: Ein Rekonstruktionsversuch". Kölner Zeitschrift für Soziologie und Sozialpsychologie, 33: 397-436.
Flora, P./J. Alber, 1981: Modernization, Democratization, and the Development of Welfare States in Western Europe". pp. 37-80 in: P. Flora/A. J. Heidenheimer (eds.), The Development of Welfare States in Europe and America. New Brunswick: Transaction Books.

Freeman, R. A., 1981: The Wayward Welfare State. Stanford: Hoover Press.

Frieden, J., 1981: „Third World Indebted Industrialization: International Finance and State Capitalism in Mexico, Brazil, Algeria, and South Korea". International Organization. 35: 407-431.

Georgescu-Roegen, N., 1975: „Energy and Economic Myths". Southern Economic Journal, 41: 347-381.

Gerschenkron, A., 1962: Economic Backwardness in Historical Perspective. Cambridge, Mass.: Harvard University Press.

Gershuny, J. I., 1978: After Industrial Society? The Emerging Self-Service Economy. London: MacMillan.

Gershuny, J. I. and I. D. Miles, 1983: The New Service Economy. The Transformation of Employment in Industrial Societies. New York: Praeger.

Gilpin, R., 1975: U. S. Power and the Multinational Corporation. New York: Basic Books.

Glyn, A./B. Sutcliffe, 1972: British Capitalism, Workers and the Profit Squeeze. Harmondsworth: Penguin.

Gourevitch, P. A., 1977: „International Trade, Domestics Coalitions, and Liberty: Comparative Responses to the Crisis of $1877-1896^{\circ .}$. Journal of Interdisciplinary History, 8: 281-313.

Gourevitch, P. A., 1978: „The Second Image Reversed: The International Sources of Domestic Politics". International Organization, 32: 881-911.

Gourevitch, P. A., 1984: „Breaking with Orthodoxy: The Politics of Economic Policy Responses to the Depression of the 1930s“. International Organization. 38: 95-129.

Grew, R., 1978: (ed.) Crises of Political Development in Europe and the United States. Princeton, N. J.: Princeton University Press.

Grew, R., 1978a: „The Crises and Their Sequences“, pp. 3-39 in Grew 1978a.

Grossman, S. J./J. E. Stiglitz, 1980: „On the Impossibility of Informationally Efficient Markets“. American Economic Review, 70: 393-408.

Habermas, J., 1973: Legitimationsprobleme im Spätkapitalismus. Frankfurt/Main: Suhrkamp.

Habermas, J., 1976: Rekonstruktion des Historischen Materialismus. Frankfurt/Main: Suhrkamp.

Habermas, J., 1981: Theorie des kommunikativen Handelns. Bd. 2. Frankfurt/Main: Suhrkamp.

Hanrieder, W. F., 1978: „Dissolving International Politics: Reflections on the Nation-State“. American Political Science Review, 72: 1276-1287.

Hauff, V. und F. W. Scharpf, 1975: Modernisierung der Volkswirtschaft. Frankfurt/Main: Europäische Verlagsanstalt.

Heclo, H., 1974: Modern Social Policies in Britain and Sweden. New Haven: Yale University Press. 
Heclo, H., 1978: "Issue Networks and the Executive Establishment". pp. 87-124 in: A. King (ed.) The New American Political System. Washington, D. C.: American Enterprise Institute.

Heclo, H., 1981: „Toward a New Welfare State?“, pp. 383-406 in: P. Flora and A. Heidenheimer (eds.) The Development of Welfare States in Europe and America. New Brunswick: Transaction Books.

Heidenheimer, A. J./H. Heclo/C. Teich-Adams, 1983: Comparative Public Policy. New York: St. Martin's Press.

Hilton, R. (ed.), 1976: The Transition from Feudalism to Capitalism. London: New Left Books.

Hirsch, F., 1976: The Social Limits of Growth. Cambridge, Mass.: Harvard University Press.

Hirsch, J., 1980: Der Sicherheitsstaat. Frankfurt/Main: Europäische Verlagsanstalt.

Hirschman, A. O., 1981: Shifting Involvements. Private Interests and Public Action. Princeton, N. J.: Princeton University Press.

Hirshleifer, J./J. G. Riley, 1979: „The Analytics of Uncertainty and Information. An Expository Survey". Journal of Economic Literature, 17: 1375-1421.

Hobsbawm, E. J., 1981: „Die Krise des Kapitalismus in historischer Perspektive“. S. 35-52 in: F. Fröbel/J. Heinrichts/O. Kreye (Hrsg.) Krisen in der kapitalistischen Weltökonomie. Reinbek: Rowohlt.

Huber, J., 1980: „Ökologische Wirtschaft“. S. 321-355 in: G. Michelsen/F. Kaberlah (Hrsg.), Der Fischer Öko-Almanach. Frankfurt/Main: Fischer.

Huber, J., 1982: Die verlorene Unschuld der Ökologie. Reinbek: Rowohlt.

Inglehart, R., 1977: The Silent Revolution. Changing Values and Political Styles Among Western Publics. Princeton, N. J.: Princeton University Press.

Institute for Contemporary Studies (ed.), 1976: The Politics of Planning. A Review and Critique of Centralized Economic Planning. San Francisco: Institute for Contemporary Studies.

Jenkins, J. C., 1981: „Sociopolitical Movements“. pp. 81-153 in: S. L. Long (ed.), Handbook of Political Behavior, 4, New York: Plenum Press.

Jessop, B., 1980: „The Transformation of the State in Post-War Britain“. pp. 23-93. R. Scase (ed.), The State in Western Europe. New York: St. Martin's Press.

Johnson, W., 1979: Muddling Toward Frugality. A Blueprint for Survival in the 1980s. Boulder: Shambala Books.

Katzenstein, P. (ed.), 1978: Between Power and Plenty. Madison: University of Wisconsin Press.

Keohane, R./J. S. Nye, 1977: Power and Interdependence. Boston: Little, Brown.

Kitschelt, H., 1983a: Politik und Energie. Energie-Technologiepolitiken in den USA, der Bundesrepublik Deutschland, Frankreich und Schweden. Frankfurt/ Main: Campus.

Kitschelt, H., 1983b: Policy Impacts of Anti-Nuclear Movements. Comparative Perspectives. Paper deliver- ed at the Fourth International Conference of Europeanists, Washington, D. C., October 13-15.

Kitschelt, H., 1984a: Der ökologische Diskurs. Frankfurt/Main: Campus.

Kitschelt, H., 1984b: „New Social Movements in West Germany and the United States". Political Power and Social Theory, 5 (im Erscheinen).

Kochen, M./K. W. Deutsch, 1980: Decentralization. Sketches Toward a Rational Theory. Cambridge, Mass.: Oelgeschlager, Gunn and Hain.

Kohl, J., 1981: „Trends and Problems in Postwar Public Expenditure Development in Western Europe and North America“. pp. 308-344 in P. Flora/A. J. Heidenheimer (eds.) The Development of Welfare States in Europe and North America. New Brunswick: Transaction Books.

Krasner, S. D., 1984: „Review Article: Approaches to the State. Alternative Conceptions and Historical Dynamics“. Comparative Politics, 17: 223-246.

Kudrle, R. T./T. R. Marmor, 1981; „The Development of Welfare States in North America“. pp. 81-121. P. Flora/A. J. Heidenheimer (eds.) The Development of Welfare States in Europe and North America. New Brunswick: Transaction Books.

Kurth, J. R., 1979a: „Industrial Change and Political Change: A European Perspective“", pp. 319-362 in: D. Collier (ed.), The New Authoritatianism in Latin America. Princeton, N. J.: Princeton University Press.

Kurth, J. R., 1979b: „The Political Consequences of the Product Cycle: Industrial History and Political Outcomes". International Organization, 33: 1-34.

Lane, R. E., 1978: „Markets and the Satisfaction of Human Wants“. Journal of Economic Issues, 12: 799-827.

Lange, P./G. Garrett, 1985: „The Organizational and Political Determinants of Economic Performance, 1974-1980“. Journal of Politics (i. V.)

Lehner, F./K. Schubert/B. Geile, 1984: „Die strukturelle Rationalität regulativer Politik". Politische Vierteljahresschriften, 24: 361-384.

Lenhardt, G., 1980: „Theorie der Rationalisierung und Sozialismuskritik bei Max Weber". Leviathan, 8: 295-319.

Leiss, W., 1976: The Limits of Satisfaction. Toronto: Toronto University Press.

Lindberg, L. N., 1976: „Strategies and Priorities for Comparative Research“. pp. 222-286 in: ders (ed.) Politics and the Future of Industrial Societies. New York.

Lipset, S. M. and S. Rokkan, 1967: „Cleavage Structures, Party Systems, and Voter Alignments: An Introduction“. in: dies. (eds.), Party Systems and Voter Alignments. New York: Free Press.

Luhmann, N., 1975: „Evolution und Geschichte“. S. 150-169 in ders., Soziologische Aufklärung, Bd. II. Opladen: Westdeutscher Verlag.

Marshall, T. H., 1949: "Citizenship and Social Class". pp. 71-134 in: ders., Class, Citizenship and Social Development. Chicago: University of Chicago Press, 1977. 
Mc Carthy, J. D./M. N. Zald, 1977: „Resource Mobilization and Social Movements: A Partial Theory". American Journal of Sociology, 82: 1212-1241.

Meixner, H., 1980: Technologische Entwicklung und natürliche Reproduktionsgrundlage. Frankfurt/Main: Haag und Herchen.

Melucci, A., 1980: „The New Social Movements: A Theoretical Approach". Social Science Information, 19: 199-226.

Melucci, A., 1981: „Ten Hypotheses for the Analysis of New Movements". pp. 173-194 in: D. Pinto (ed.), Contemporary Italian Sociology. Cambridge, Mass.: Cambridge University Press.

Mitnick, B. M., 1980: The Political Economy of Regulation. New York: Columbia University Press.

Moore, B., Jr., 1966: Social Origins of Dictatorship and Democracy. Boston: Beacon Press.

Moscivici, S., 1977: „The Re-enchantment of the World". pp. 131-168 in: N. Birnbaum (ed.), Beyond the Crisis. London: Oxford University Press.

Müller-Rommel, F., 1983: „Die Postmaterialismusdiskussion in der empirischen Sozialforschung: Politisch und wissenschaftlich überlebt oder noch immer zukunftsweisend?" Politische Vierteljahresschrift, Bd. 24: 218-228.

Naschold, F., 1981: „Developmental Crises and Modernization: The Role of the State in the Redistribution Crisis in Developed Countries“. pp. 115-142 in: R. L. Merritt/B. M. Russett (eds)., From National Development to Global Community. London: Allen and Unwin.

North, D. C., 1981: Structure and Change in Economic History. New York: Norton.

North, D. C./R. Thomas, 1973: The Rise of the Western World. Cambridge: Cambridge University Press.

O'Connor, J., 1973: Die Finanzkrise des Staates. Frankfurt/Main: Suhrkamp.

Offe, C., 1969: „Politische Autorität und Klassenstrukturen“. S. 135-164 in: G. Kress/D. Senghaas (Hrsg.), Politikwissenschaft. Frankfurt/Main: Fischer.

Offe, C., 1973: „,Krisen des Krisenmanagements‘. Elemente einer politischen Krisentheorie“. S. 197-223 in: Jänicke (Hrsg.), Herrschaft und Krise. Opladen: Westdeutscher Verlag.

Offe, C., 1974: „Rationalitätskriterien und Funktionsprobleme politisch-administrativen Handelns“. Leviathan. 2: 333-345.

Offe, C., 1980: „Konkurrenzpartei und politische Identität“. S. 26-42 in: R. Roth (Hg.) Parlamentarisches Ritual und politische Alternativen. Frankfurt/Main: Campus.

Offe, C., 1981: „The Attribution of Public Status to Interest Groups: Observations on the West German Case“. pp. 123-158 in: S. Berger (ed.), Organizing Interests in Western Europe. Cambridge, Mass.: Cambridge University Press.

Offe, C., 1983: „Competitive Party Democracy and the Keynesian Welfare State: Factors of Stability and Disorganization“. Policy Sciences, 15: 225-246.
Olson, M., Jr., 1982: The Rise and Decline of Nations. New Haven: Yale University Press.

Ophuls, W., 1977: Ecology and the Politics of Scarcity. San Francisco: Freeman.

Parsons, T./N. Smelser, 1956: Economy and Society. New York: Free Press.

Piore, M./C. Sabel, 1985: The Second Industrial Divide. New York: Basic Books (in Vorbereitung).

Pizzorno, Allessandro, 1981: „Interests and Parties in Pluralism“. pp. 247-284 in: S. Berger (ed.), Organizing Interests in Western Europe. Cambridge, Mass.: Cambridge University Press.

Polanyi, Karl, 1957: The Great Transformation. Boston: Beacon Press.

Posner, R. A., 1981: „The Economics of Privacy“. American Economic Review, Papers and Proceedings. 71: 399-404.

Poulantzas, N., 1975: Klassen im Kapitalismus heute. Hamburg: VSA.

Rifkin, J., 1980: Entropy. A New World View. New York: Viking Press.

Rokkan, S., 1970: Citizens, Elections, Parties. New York: David McKay.

Rokkan, S., 1975: „Dimensions of State Formation and Nation Building. A Possible Paradigm for Research on Variations within Europe“. pp. 562-600 in Tilly (ed.), 1975a.

Ronge, V., 1975: „Entpolitisierung der Forschungspolitik“. Leviathan, 3: 307-337.

Rosier, B./P. Dockès, 1983: Rythmes économiques. Crises et changement social. Une perspective historique. Paris: Maspero.

Ruggie, J. G., 1975: „International Responses to Technology: Concepts and Trends". International Organization. 29: 557-583.

Sabatier, P. A., 1975: „Social Movements and Regulatory Agencies: Toward a More Adequate and Less Pessimistic Theory of ,Clientele Capture'. " Policy Sciences, 6: 301-342.

Sabel, C., 1982: Work and Politics. Cambridge, Mass.: Cambridge University Press.

Schefold, B., 1977: „Energy and Economic Theory“. Zeitschrift für Wirtschafts- und Sozialwissenschaft, 7 : 227-249.

Schmid, M., 1981: „Struktur und Selektion: Emile Durkheim und Max Weber als Theoretiker struktureller Selektion“. Zeitschrift für Soziologie, 10: 17-37.

Schmidt, M. G., 1982: Wohlfahrtsstaatliche Politik unter bürgerlichen und sozialdemokratischen Regierungen. Frankfurt/Main: Campus.

Schmidt, M. G., 1983: „The Welfare State and the Economy in Periods of Economic Crisis: A Comparative Study of 23 OECD Nations“. European Journal of Political Research, 11: 1-26.

Schmitter, P. C., 1981: „Interest Intermediation and Regime Governability in Contemporary Western Europe and North America". pp. 287-330 in: S. Berger (ed.), Organizing Interests in Western Europe. Cambridge, Mass.: Cambridge University Press. 
Schumpeter, J. A., 1975: Capitalism, Socialism, and Democracy. New York: Harper.

Scitovsky, T., 1976: The Joyless Economy. New York: Oxford University Press.

Semmler, W., 1982: „Economic Aspects of Model Germany: A Comparison wite the United States". pp. 22-52 in: A. S. Markovits (ed.) The Political Economy of West Germany. Modell Deutschland. New York: Praeger.

Shonfield, A., 1965: Modern Capitalism. London: Oxford University Press.

Siebert, H. (Hrsg.), 1980: Erschöpfbare Ressourcen. Berlin: Duncker und Humblot.

Simonnet, D., 1979: L'écologisme. Paris: Presses universitaires de France.

Skocpol, T., 1979: States and Revolutions. Cambridge, Mass.: Cambridge University Press.

Smith, A. D., 1973: The Concept of Social Change. A Critique of the Functionalist Theory of Social Change. London: Routledge and Kegan Paul.

Strasser, J./K. Traube, 1981: Die Zukunft des Fortschritts. Der Sozialismus und die Krise des Industrialismus. Bonn: Verlag Neue Gesellschaft.

Thompson, W. R., 1983: „The World Economy, the Long Cycle, and the Question of World System Time“. pp. 35-62 in: P. McGowan/C. W. Kegley, Jr. (eds.), Foreign Policy and the Modern World System. Beverly Hills: Sage.

Tilly, C., 1975a: (ed.) The Formation of National States in Western Europe. Princeton, N. J.: Princeton University Press.

Tilly, C., 1975b: „Reflections on the History of European State Making“. pp. 3-83 in Tilly, 1975a.
Tilly, C., 1978: From Mobilization to Revolution. Reading, Mass.: Addison-Wesley.

Touraine, A., 1971: The Post-Industrial Society. New York: Random House.

Touraine, A., 1977a: The Self-Production of Society. Chicago: University of Chicago Press.

Touraine, A., 1977b: „Crisis or Transformation?“, pp. 17-45 in: Norman Birnbaum (ed.), Beyond the Crisis. London: Oxford University Press.

Touraine, A., 1978: La voix et le regard. Paris: Seuil.

Van Parijs, P., 1982: „Functionalist Marxism Rehabilitated". Theory and Society, 11: 497-511.

Verba, S., 1971: "Sequences and Development". pp. 283-316 in Binder, 1971.

Vobruba, G., 1978: „Staatseingriff und Ökonomiefunktion. Der Sozialstaat als Problem für sich selbst". Zeitschrift für Soziologie, 7: 130-156.

Wallerstein, I., 1974: The Modern World System. New York: Academic Press.

Warner, R. S., 1978: „Toward a Redefinition of Action Theory: Paying the Cognitive Element its Due“. American Journal of Sociology. 83: 1317-1349.

Weisskopf, T. E., 1981: „The Current Economics Crisis in Historical Perspective". Socialist Review, 11: 9-53.

Williamson, O. E., 1975: Markets and Hierarchies. New York: Free Press.

World Bank, 1982: World Development Report. London: Oxford University Press.

Yergin, D./M. Hillenbrand (eds.), 1982: Global Insecurity. A Strategy for Energy and Economic Renewal. Boston: Houghton Mifflin.

Zysman, J., 1983: Governments, Markets, and Growth. Ithaca, N. Y.: Cornell University Press. 